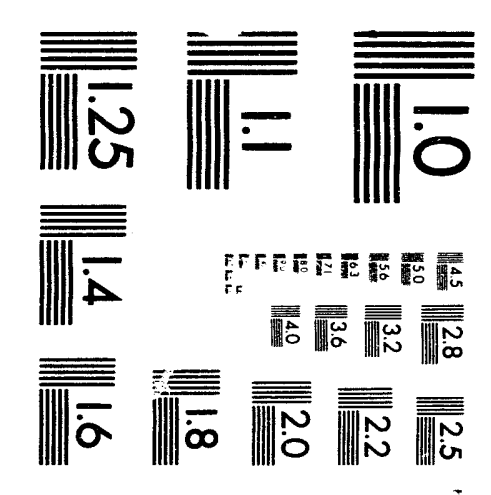



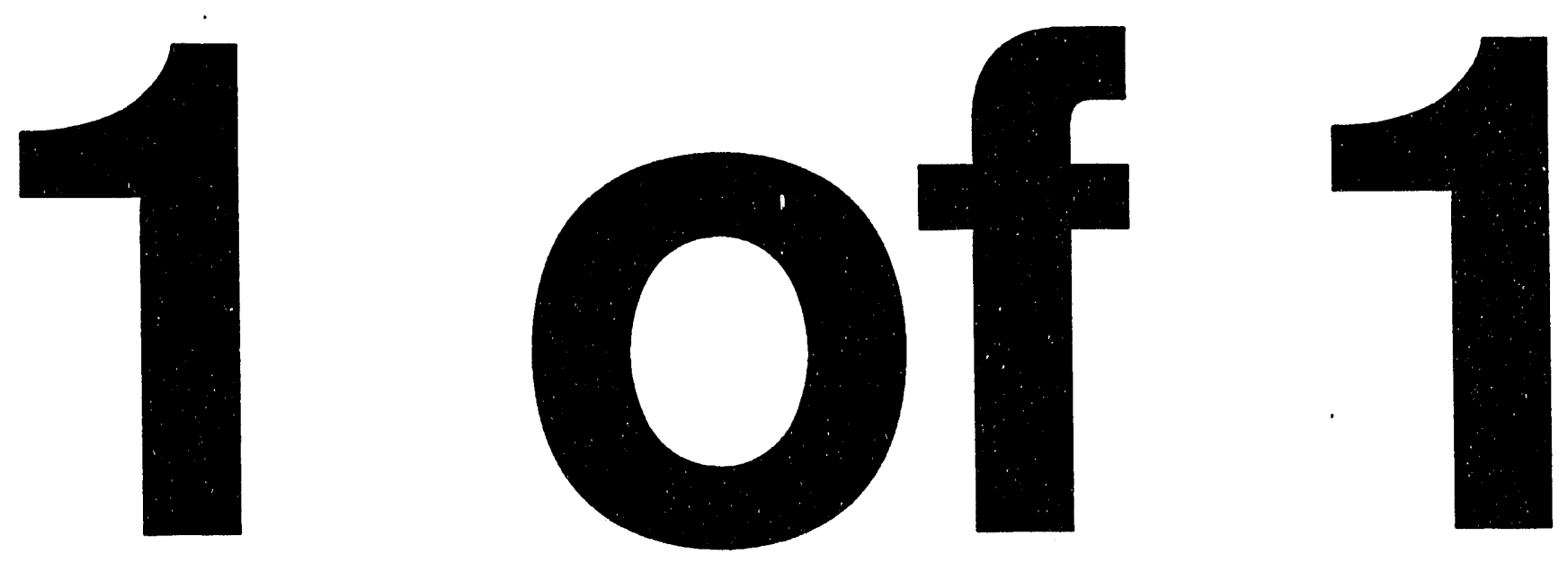
DOE/ER/40592-3

\title{
Quantum Chromodynamics
}

and

\section{Nuclear Physics at Extreme Energy Density}

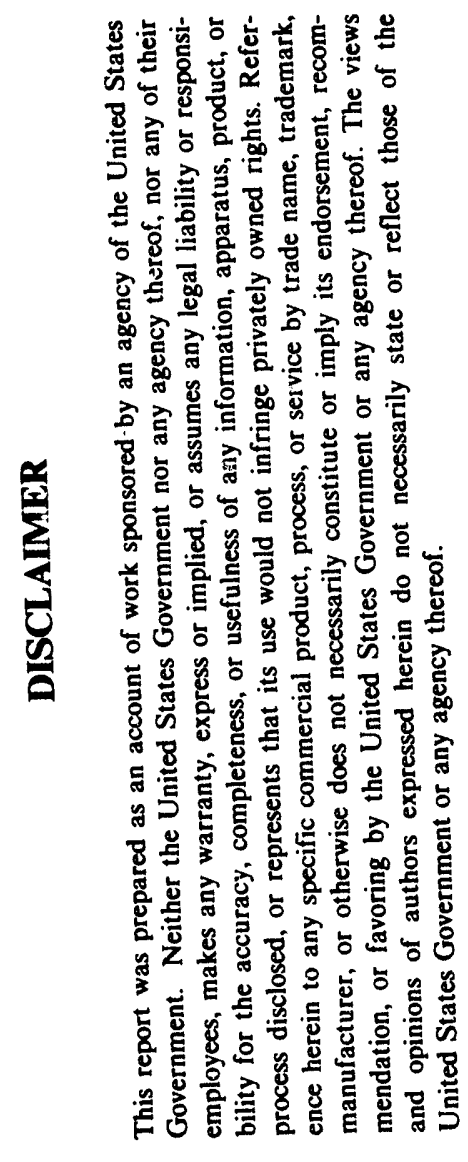

\author{
Progress Report \\ May 1992 - April 1993 \\ BERNDT MÜLLER \\ ROXANNE P. SPRINGER \\ Department of Physics \\ Duke University \\ Durham, NC 27708-0305
}

15 May 1993

Prepared for the U.S. Department of ENERgy

UNDER Grant Number DE-FG-5-90ER40592

\section{MASTER}

ustupuring of rels nacusant is unumrra 


\section{TABLE OF CONTENTS}

1. Hadron Structure Physics 1

1.1. Strong Decays of the Baryon Decuplet 1

1.2. Radiative Decays of the Baryon Decuplet 3

1.3. Some Comments on Heavy Baryon Chiral Perturbation Theory 6

1.4. Strange Hadronic Matter 7

2. Relativistic Heavy Ion Collisions 10

2.1. Medium Modifications of Parton Cross Sections 10

2.2. Parton Equilibration 13

2.3. Multiply Collisions Induced Gluon Bremsstrahlung in QCD 16

$\begin{array}{lll}\text { 2.4. Branching Processes in Multiparticle Production } & 17\end{array}$

3. Finite-Temperature QCD 18

3.1. Magnetic Screening in Thermal Yang-Mills Theories 18

3.2. Magnetic Field Correlator on the Lattice 21

3.3. The $\lambda \phi^{4}$ Theory on a Four-dimensional Torus 22

4. Real-Time Lattice Gauge Theory 25

4.1. Lyapunov Exponent of SU(2) Gauge Theory 25

4.2. Classical Nature of Gluon Damping Rate 27

4.3. Lyapunov Spectra in Gauge Theory by Rescaling Method 28

4.4. Chaoticity in Massless Higgs Fields and Massive Gauge Fields 30

4.5. Gaussian Wavepacket and Quantum Corrections in SU(2) 32

4.6. Thermalization in SU(2) Gauge Theory 35

4.7. Real-Time Evolution in the Non-Linear Sigma Model 38

5. Studies In Quantum Field Theory 41

5.1. Irreversibility in Quantum Theory 41

5.2. Entropy Production in Vacuum Decay 42

5.3. Is Particle Creation Irreversible? 44

5.4. Vacuum Polarization and Electric Charge of the Positron 46

$\begin{array}{ll}6 . & 49\end{array}$

$\begin{array}{ll}\text { I. Publications } & 49\end{array}$

II. Lectures and Seminars $\quad 50$

III. Triangle Nuclear Theory Colloquium 52

IV. Theory Seminars at Duke 54 


\section{Hisdon Structure Physics}

A study of the properties of the decuplet of baryons shows great promise for illuminating nonperturbative aspects of Quantum Chromodynamics. As one of the key areas of physics that remains to be understood, nonperturbative properties of QCD may dictate the binding of quarks and gluons into nucleons. Currently, perturbative calculations work well as a model-independent means of determining quark-level interactions at high energy (where $\alpha_{s} \ll 1$ ). Yet, to relate these calculations to experimental measurements, results are needed in terms of hadronic degrees of freedom. Progress on the lattice front is forthcoming; what is needed at this point is a theory that can connect lattice results to perturbative results to provide complete predictive power over the range of energy necessary for reconciling and explaining the QCD symmetries and the resulting hadrons. Only then can we say we have complete understanding of the nucleon.

It is an extremely relevant time for such a study because of the status of the Continuous Electron Beam Accelerator Facility; there will soon be experimental data of such detail on the decuplet of baryons that a discrimination amongst effective theories can be made. The technique used here is that of the Heavy Baryon Chiral Perturabtion Theory (HBChPT) [1]. Details are given in the reference, the important points being that this is a theory which is a consequence of the symmetries of QCD and which encorporates a systematic expansion in powers of small momenta or light quark mass over the chiral breaking scale or baryon mass. The fundamental fields are the hadrons themselves, both the octet of light mesons and the octet and decuplet of baryons, circumventing the usual difficulties associated with the approximations that must be made to find hadronic matrix elements of quark-level hamiltonians. Further, calculations done in this theory can be used to explore how (or whether) the symmetries of QCD survive in the hadronic system. This is currently of great interest, considering the somewhat inexplicable usefulness of an $\mathrm{SU}(6)$ spin-flavor symmetry for phenomenological predictions [2].

\subsection{Strong Decays of the Baryon Decuplet}

(R. P. Springer $\left[{ }^{*}\right]$ )

As a first step towards implimenting the above discussion, we consider the loop corrections associated with meson emission from baryons. The Lagrangian governing the behavior of the octet baryons with the octet mesons is, to lowest order,

$$
\mathcal{L}_{v}^{8}=i \operatorname{Tr} \bar{B}_{v}(v \cdot \mathcal{D}) B_{v}+2 D \operatorname{Tr} \bar{B}_{v} S_{v}^{\mu}\left\{A_{\mu}, B_{v}\right\}+2 F \operatorname{Tr} B_{v} S_{v}^{\mu}\left[A_{\mu}, B_{v}\right] .
$$

Here $B_{v}$ is the $3 \times 3$ matrix of octet baryons whose fields have been redefined to remove explicit baryon masses; they have a definite velocity, $v$. The covarient chiral derivative, $\mathcal{D}_{\mu}=\partial_{\mu}+\left[V_{\mu},\right]$, is given in terms of

$$
V^{\mu}=\frac{1}{2}\left(\xi D^{\mu} \xi^{\dagger}+\xi^{\dagger} D^{\mu} \xi\right)
$$

where

$$
D_{\mu}=\partial_{\mu}+i \mathcal{A}_{\mu}[Q,]
$$


contains the electromagnetic field, $\mathcal{A}$, and the $\mathrm{SU}(3)$ charge operator, $\mathrm{Q}$. The octet of boson fields, the $3 \times 3$ matrix $M$, is included through

$$
\xi=\exp \left(i M / f_{\pi}\right),
$$

where $f_{\pi}$ is the mesonic decay constant. The decuplet of baryons, $T$, is included through the terms in

$$
\begin{aligned}
\mathcal{L}_{v}^{10}= & -i \bar{T}_{v}^{\mu}(v \cdot \mathcal{D}) T_{v \mu}+\Delta m \bar{T}_{v}^{\mu} T_{v \mu}+\mathcal{C}\left(\bar{T}_{v}^{\mu} A_{\mu} B_{v}+\bar{B}_{v} A_{\mu} T_{v}^{\mu}\right) \\
& +2 \mathcal{H} \bar{T}_{v}^{\mu} S_{v \nu} A^{\nu} T_{v \mu} .
\end{aligned}
$$

The fields are redefined as before, only now the appearance of $\Delta m$, the mass splitting between the decuplet and the octet resonances, is required. Both (1.1) and (1.4) are defined in terms of a spin operator, $S_{v \nu}$, acting on the baryon fields, and the vector field,

$$
A^{\mu}=\frac{i}{2}\left(\xi D^{\mu} \xi^{\dagger}-\xi^{\dagger} D^{\mu} \xi\right) .
$$

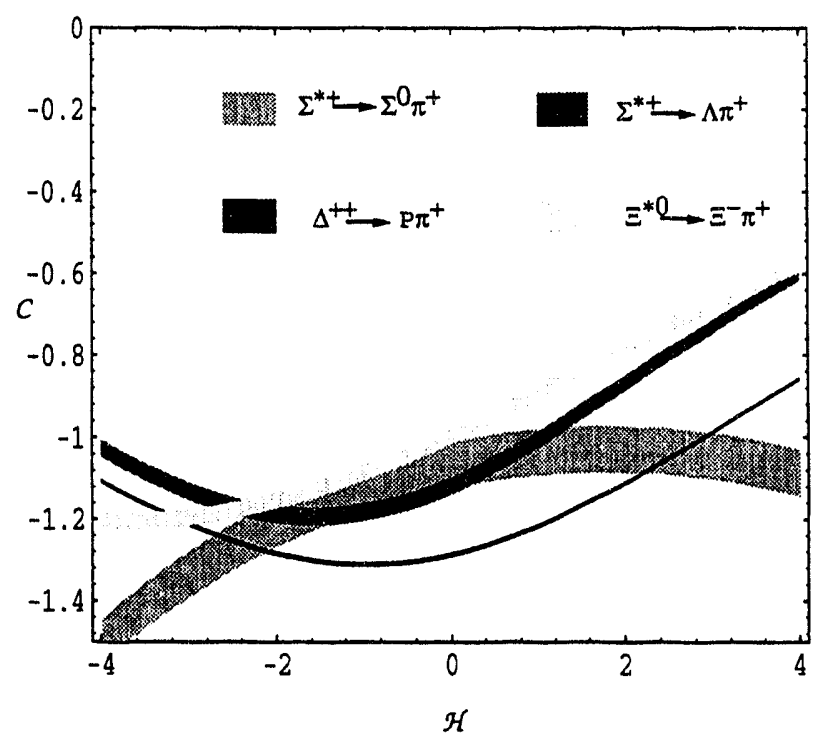

Fig.1: The decuplet-octet-meson coupling constant $\mathcal{C}$ as a function of the decuplet-decuplet-meson coupling constant $\mathcal{H}$ for the decays $\Delta^{++} \rightarrow$ $p \pi^{+}, \Sigma^{*+} \rightarrow \Sigma^{0} \pi^{+} \rightarrow \Lambda \pi^{+}$and $\Xi^{*+} \rightarrow \Xi^{-}-\pi^{+}$. The width of each line represents the $1 \sigma$ error arising from experimental determinations of the baryon resonant width. There is no theoretical uncerainty included in the curves. Note that the sign of a $\mathcal{C}$ is chosen for ease of comparison to $\mathrm{SU}(6)$ predictions, but is in fact undetermined in $\mathrm{HBChPT}$.

At this point, the strong interaction coupling constants are $F$ and $D$ amongst the octets, and the strong decay of the decuplet is characterized by $\mathcal{C}$. Taken directly from the Lagrangian, a tree level comparison to experimental decay rates can be made, and 
a value for $\mathcal{C}$ extracted. The tree-level calculation is necessarily $\mathrm{SU}(3)$ conserving, while the experimental results obviously are not. Including the leading nonanalytic terms which result from kaon loops will allow a more accurate extraction of $\mathcal{C}$ from experimental data. The result is given in Fig 1 . Note how $\mathcal{C}$ and $\mathcal{H}$ become nearly universal for the four decay rates in the range $1.1<|\mathcal{C}|<1.3$ and $-2.8<\mathcal{H}<-1.6$. This range of $\mathcal{H}$ agrees well with the constraint found in [3]. In particular, the results found here, along with those found in [4] show that the $\mathrm{SU}(6)$ relationships,

$$
\begin{aligned}
& F=\frac{2}{3} D \quad \mathcal{C}=-2 D \\
& \mathcal{H}=-3 D .
\end{aligned}
$$

are well satisfied in chiral perturbation theory; an interesting circumstance since the theory is based on the $\mathrm{SU}(3)$ of $\mathrm{QCD}$ alone.

[*] M. Butler, M. Savage and R. Springer, Nucl. Phys. B (in press).

[1] E. Jenkins and A. Manohar, Proceedings of the Workshop on "Effective Field Theories of the Standard Model", ed. U. Meissner, World Scientific (1992).

[2] C. Carone and H. Georgi, Nucl. Phys. B375 (1992) 243.

[3] E. Jenkins and A. Manohar, Phys. Lett. 255B (1991) 558; Phys. Lett. 259B (1991) 353.

[4] E. Jenkins, Nucl. Phys. B368 (1992) 190; E. Jenkins, Nucl. Phys. B375 (1992) 561.

\subsection{Radiative Decays of the Baryon Decuplet}

\section{(R. P. Springer $\left[{ }^{* *}\right]$ )}

The electromagnetic decays of the decuplet provide another area where the theory of chiral lagrangians can be tested, and further insights into the properties of these resonances can be obtained. In particular, the ratio of electric quadrupole to magnetic dipole transition rates is still quite controversial. Unfortunately, it appears that all experimental extractions
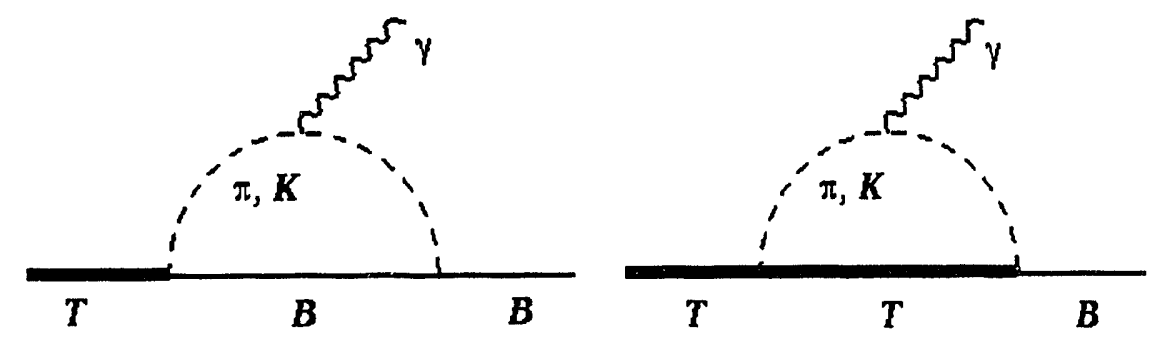

Fig. 2: Graphs contributing to the radiative decay of decuplet baryons. The heavy line represents a decuplet baryon, the solid line an octet baryon, the dashed line an octet meson, and the wavy line a photon. We work in $\epsilon_{\gamma} \cdot v=0$ gauge where there is no direct coupling of the photon to either the octet or decuplet baryon. 
to date are model-dependent [5-8], which further complicates the issue. The one loop graphs responsible for the decay rate in $\mathrm{HBChPT}$ are shown in Fig 2. Unlike the case for strong decays, a counterterm exists for the radiative decays whose coefficient must be fixed by one of the measured decuplet decay rates (the $\Delta$ particle is used for this). Our results appear in Table 1. Note that the SU(3) forbidden decays, given in the last two rows, are independent of the above mentioned counterterm and thus are a cleaner test of the effects of kaon loops, and the nature of $\mathrm{SU}(3)$ violating loop corrections in HBChPT. Again, we hope that CEBAF will measure these decay rates soon. In addition, some recent lattice work [9] shows agreement with our results.

\section{Table 1: Decuplet Branching Ratios (\%)}

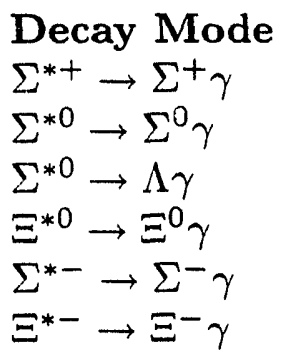

Branching Ratio (\%)
$0.2-0.6$
$0.04-0.1$
$0.8-1.3$
$1.0-3.0$
$0.004-0.006$
$0.01-0.03$

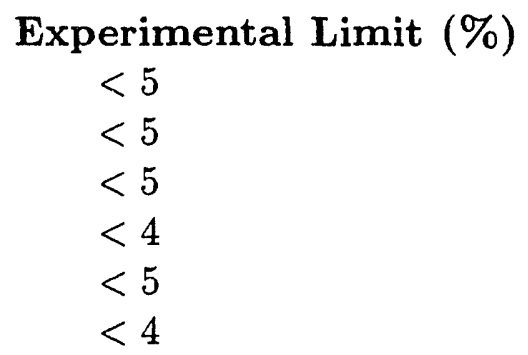

One way of investigating the internal structure of the decuplet hadrons is to study the ratio of the electric quadrupole to magnetic dipole radiation, E2/M1, emitted by the hadron. There is experimental information on this ratio from the decay $\Delta \rightarrow N \gamma$, which gives us an opportunity to make comparisons for at least this mode. As noted above, a recurring theme in the study of the decuplet is how well $\mathrm{HBChPT}$ seems to reproduce exactly those results that might be expected from an SU(6) spin-flavor symmetry. To investigate this further, in an attempt to find the source of the appearance of this symmetry, we study a process that is very much at odds with the simplest constituent quark model; the $\mathrm{E} 2 / \mathrm{M} 1$ ratio. Another reason this ratio is interesting for $\mathrm{HBChPT}$ is that the two multipoles have such different sources within the theory. The M1 amplitude is mainly a short-distance phenomenon, the main ingredients being the counterterm introduced above, and the contribution of kaon loops. The $\mathrm{E} 2$ amplitude, in contrast, is very much a long distance effect, with long-distance pion loops the dominant contribution. The later can be calculated with some confidence in the chiral theory. Results for two of the processes are given in Fig. 3. Note that there is an imaginary part to this ratio since the final state interactions between octet baryon and pion with a decuplet baryon occur. The importance of what we have done here lies in the inclusion of the long-distance, pionic effects, which appear to have much significance in the ratio. Lattice calculations have been done to find this ratio [9], yet have no imaginary part since the decuplet particles are taken to be stable. The darker region on the plots shown indicates the prediction found when the SU(6) relationships amongst the chiral langrangian coupling constants is imposed. An experimental determination of the ratios will provide a strict test for this symmetry, yielding a further understanding of the validity of this approach.

In an effort to understand pion photoproduction, where experimental tests are currently available, we look at the radiative decay of the delta which ends in a pair of leptons 
as the final state. The radiative decay discussed earlier probes the hadronic structure of the decuplet of baryons, yet is restricted to the case where the emitted photon is on its mass shell. With a leptonic final state, we have access to the off-shell behavior of the radiative coupling and can look at the momentum transfer dependence of the E2/M1 ratios. The result is given as a function of the invarient mass of the lepton pair, as shown in Fig. 4. Experimental measurements of these decays are expected from CEBAF, and a comparison with the results from HBChPT should be important in understanding the ground state structure of the baryons.
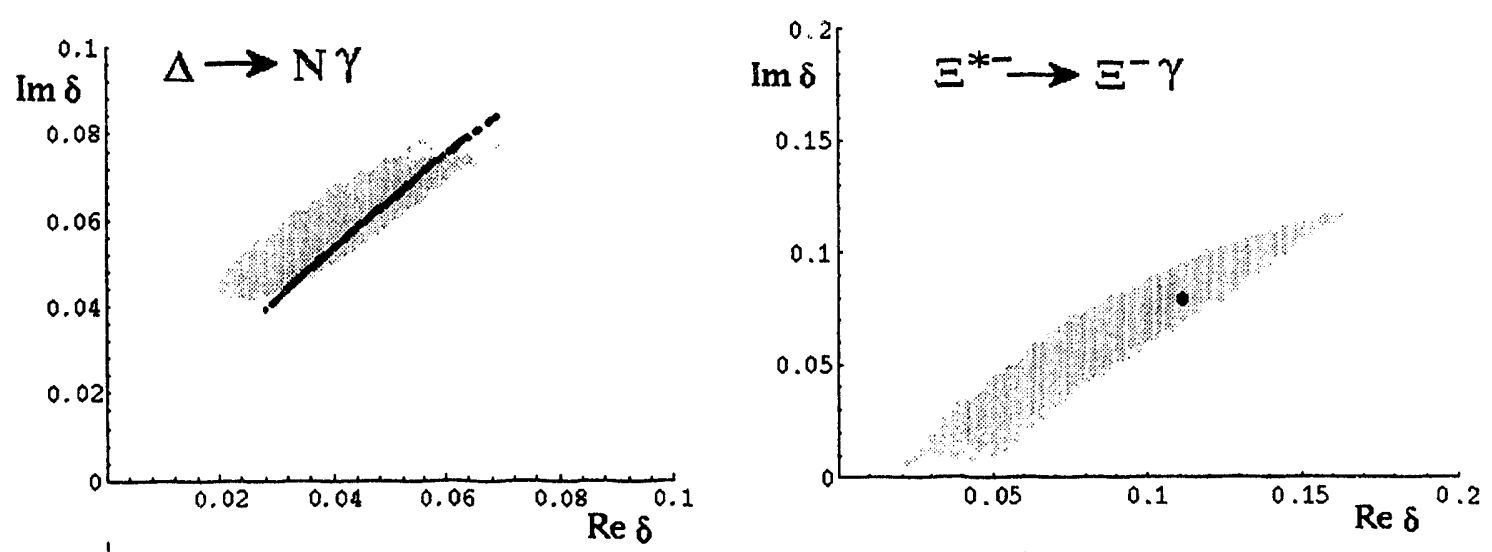

Fig. 3: The $\mathrm{E} 2 / \mathrm{M} 1$ ratio $\left(\delta=\delta_{\mathrm{E} 2 / \mathrm{M} 1}\right)$ for the $\mathrm{SU}(3)$ allowed radiative decay $\Delta \rightarrow N \gamma$ (left) and the $\mathrm{SU}(3)$ suppressed decay of the cascade (right). The gray region corresponds to the 6000 points randomly chosen only from the uncertainties in the couplings $F, D, \mathcal{C}, \mathcal{H}$, and the width for $\Delta \rightarrow N \gamma$. The dark line is the prediction of heavy baryon $\mathrm{SU}(6)$, chosen from the uncertainty in $D$, and still including the uncertainty in the $\Delta \rightarrow N \gamma$ width. We have assumed the E2 counterterm to be small compared to the contribution from pion loops. The dark point is the prediction of heavy baryon $\mathrm{SU}(6)$ which is independent of the choice for $D$, and independent of the $\Delta \rightarrow N \gamma$ decay width. (The suppressed decays do not receive a contribution from local counterterms.)

Finally, we are in a position to use the above results to make a comment on the contribution of the delta pole graph to nucleon polarizability [10-12]. The electric susceptibility seems to be dominated by the long-distance pion loops, while the magnetic susceptibility seems to be dominated by the $\Delta$ pole. We find that $\beta^{\text {pole }}=(2-10) \times 10^{-4} \mathrm{fm}^{3}$, which is consistent with experimental numbers.

${ }^{[* *}$ M. Butler, M. Savage, and R. Springer, preprint DUKE-TH-93-47, Phys. Lett. B (in press).

[5] H. Tanabe and K. Ohta, Phys. Rev.. C31 (1985) 1876.

[6] R. M. Davidson, N. C. Mukhopadhyay and R. S. Wittman, Phys. Rev. D43 (1991) 71.

[7] R. L. Workman, R. A. Arndt and Z. li, Virginia Polytechnic Institute and State University preprint. 
[8] S. Nozawa, B. Blankleider and T. S. H. Lee, Nucl. Phys. A513 (1990) 459.

[9] D. Leinweber, T. Draper and R. M. Woloshyn, University of Maryland Preprint, U.MD PP \#93-085.

[10] M. N. Butler and M. J. Savage UCSD/PTH 92-30, QUSTH 92-04 (1992).

[11] W. Broniowski and T. D. Cohen, University of Maryland Preprint U. of.MD PP 92193; T. D. Cohen and W. Broniowski, University of Maryland Preprint U.of.MD PP 92-191.

[12] V. Bernard et al, Preprint BUTP-92/15, CRN 92-24 (1992).

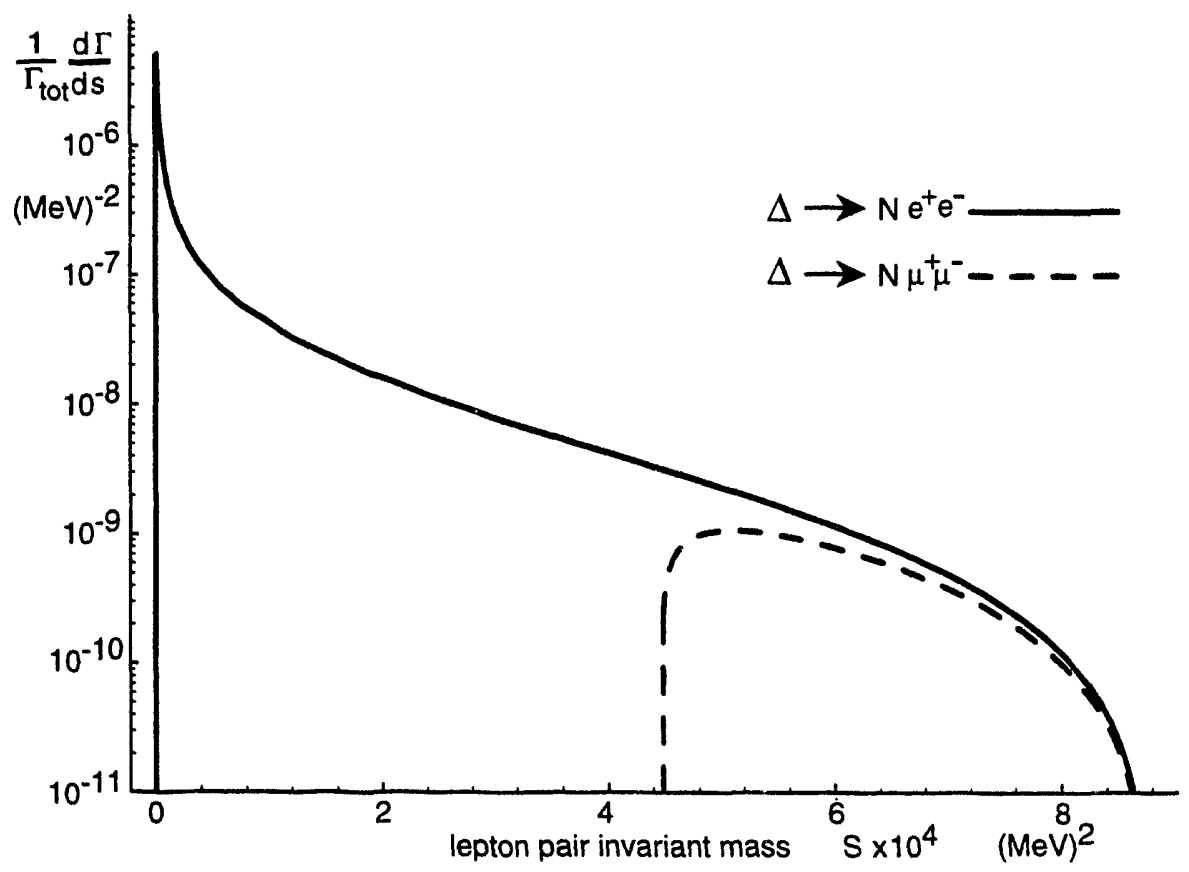

Fig. 4: Differential decay rates for the two processes $\Delta \rightarrow N e^{+} e^{-}$and $\Delta \rightarrow$ $N \mu^{+} \mu^{-}$.

\subsection{Some Comments on Heavy Baryon Chiral Perturbation Theory}

(R. P. Springer $\left[{ }^{* * *}\right]$ )

It seems relevent to discuss the accuracy to which results in HBChPT can be calculated. We have computed at the one-loop level to obtain the results discussed in sections 1.1 and 1.2. The consequent neglect of terms higher order in inverse baryon masses, derivatives, and quark masses gives rise to uncertainties of about $30 \%$. The $\mathrm{SU}(3)$ forbidden predictions tend to be more accurate, and are certainly a cleaner test for the importance of kaon loops, but are of course harder to measure experimentally. Our efforts here have been to probe hadronic structure by looking at various decay modes of the decuplet of baryons. We have applied the systematic, consistent field theory that is HBChPT to calculate model-independent results. We found that the strong decuplet decay rates select coupling constants of $1.1<|\mathcal{C}|<1.3$ and $-2.8<\mathcal{H}<-1.6$. Many of the electromagnetic decay rate predictions may be tested during the first CEBAF run. We found that the ratio 
of $\mathrm{E} 2$ to $\mathrm{M} 1$ radiation for $\Delta \rightarrow N \gamma$ falls in the range $4 \% \lesssim\left|\delta_{\mathrm{E} 2 / \mathrm{M} 1}\right| \lesssim 11 \%$, which is larger than the model-dependent estimates that have been made [13,14]. Finally, branching ratios for the decays $\Delta \rightarrow N e^{+} e^{-}$and $\Delta \rightarrow \mu^{+} \mu^{-}$are predicted at $5 \times 10^{-5}$ and $3 \times 10^{-7}$, respectively. Even for a less liberal range of experimentally accessible invarient mass, CEBAF is expected to collect important data on these modes.

We hope to understand the effect of QCD symmetries on mesons and baryons, and thereby investigate the hadronic structure of the resonances we have studied. This probes the important area of nonperturbative QCD which is likely to be crucial to our understanding of how quarks bind into hadrons. We are confident that experimental results will be obtained soon that will test the chiral theory and determine the importance of meson loops, the extent and relevance of the SU(3) symmetry breaking terms, and the limit of applicability of the SU(6) spin-flavor symmetries.

${ }^{[* * *]}$ M. Butler, M. Savage, and R. Springer, preprint DUKE-TH-93-48.

[13] S. Capstick, Phys. Rev. D46 (1992) 1965; D46 (1992) 2864.

[1.4] S. Kumano, Phys. Lett. B214 (1988) 132.

\subsection{Strange Hadronic Matter}

(C. Greiner $\left[{ }^{*}\right]$ )

Perhaps the only unambiguous way to detect the transient existence of a temporarily created quark gluon plasma might be the experimental observation of exotic remnants, like the formation of strange quark matter (SQM) droplets [1]. First studies in the context of the MIT-bag model predicted that sufficiently heavy strangelets might be absolutely stable [2] or smaller ones at least metastable [1]. The reason for the possible stability of SQM lies in introducing a third flavour degree of freedom, the strangeness, where the mass of the strange quarks is considerably smaller than the Fermi energy of the quarks, thus lowering the total mass per unit baryon number of the system. According to this picture, SQM should appear as a nearly neutral and massive state because the number of strange quarks is nearly equal to the number of massless up or down quarks and so the strange quarks neutralizes that hypothetical form of nuclear matter.

Still, on the other side, strangeness remains also a largely unexplored (experimentally as theoretically) degree of freedom in strongly interacting baryonic matter [3]. This lack of investigation reflects the experimentally task in producing nuclei containing (weakly decaying) strange baryons, which is conventionally limited by replacing one neutron (or at maximum two) by a strange $\Lambda$-particle in scattering experiments with pions or kaons.

However central relativistic heavy ion collisions provide also a source for the formation of multi-hypernuclear objects, consisting of nucleons, $\Lambda$ 's and $\Sigma$ 's. By employing a relativistic meson-baryon field theory (RMF), which gives a rather excellent description of normal nuclear and single $\Lambda$-hypernuclear properties [4], it was found that such configurations may exist as small metastable objects [5]. From a more general point of view, based on these theoretical observations one is now tempted to ask about their principle existence also as much larger objects.

For the present exploratory investigation of large multi-strange hadronic matter (SHM), two rather differnt realizations of the RMF approach have been considered, in 
order to illustrate the range of (new) phenomena which might be expected. In model 1 , an extension of the conventional scalar $(\sigma)$ and vector $(\omega)$ RMF picture to the strange sector $[4,5]$, we additionally adjust the couplings to reproduce the trend of the observed binding energies of single nucleon, $\Lambda$ and $\Sigma$ states. However, this approach results in a rather negligible weak hyperon-hyperon $(Y Y)$ interaction. To achieve a stronger interaction, as suggested by the few available $\Lambda \Lambda$ data, we further extended the RMF concept by including an additional scalar $\left(\sigma^{*} \equiv f_{0}(975)\right)$ and vector $\left(\omega^{*} \equiv \phi(1020)\right)$ meson field which couple strongly to the hyperons.

In both models we find a broad class of stable objects composed of $\left\{n, p, \Lambda, \Xi^{0}, \Xi^{-}\right\}$ baryons, with very high strangeness fraction $f_{s}=|S| / A$ (compare Fig. 1), and small net charge per baryon $Z / A \sim 0$, even negative values. These objects are stable with respect to strong decay, but can decay weakly. $\Sigma$ 's are not predicted to be sufficiently bound so as to suppress the $\Sigma N \rightarrow \Lambda N$ reaction. Interestingly, the $\Xi$ 's are populated because the corresponding reaction $\Xi N \rightarrow \Lambda \Lambda$ is Pauli blocked for a core consisting already some $\Lambda$ 's. In fact, the opposite reaction then determines the possible filling of the single-particle states.

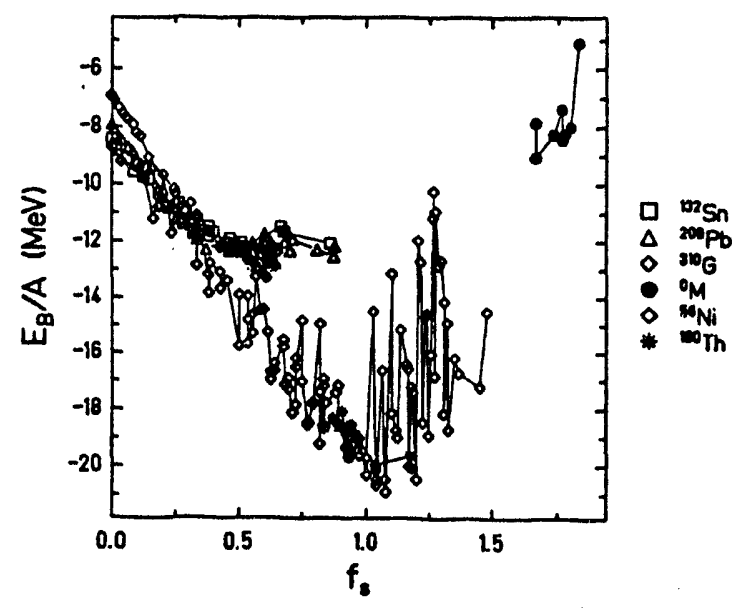

Fig. 1: The binding energy $E_{B} / A$ versus the strangeness fraction $f_{s}$ for several sequences of superstrange hypernuclei based on various nuclear cores as indicated. The calculations for the upper three cores employed model 1, i.e. weak $Y Y$ interaction, whereas the lower employed the model 2 with the stronger $Y Y$ interaction included. ${ }^{0} M$ denotes pure hypernuclei solely made out of strange baryons.

In model 1 we find binding energies as large as $E_{B} / A \approx-13 \mathrm{MeV}$ at a minimum for the strangeness content $f_{s} \approx 0.6$. Due to the stronger (and probably more realistic) $Y Y$ interaction this minimum is shifted towards a higher strangeness content $f_{s} \approx 1.1$ in model 2 , with an effective binding energy $E_{B} / A \approx-21 \mathrm{MeV}$ exceeding the value of normal nuclear matter by a few $\mathrm{MeV}$ (compare Fig. 1).

For large baryon number $\mathrm{A}$, the fission process will be removed, since the ordinary Coulomb repulsion generated by the protons can be compensated by a 
comparable number of $\Xi$ 's. Thus SHM is also to be predicted to be metastable in the bulk limit $(A \rightarrow \infty)$.

This analogy to the situation of the hypothetical SQM goes even further if one considers the baryon densities of these multihypernuclear objects, ranging from 2 to 3 times that of normal nuclear density. This is exactly the same range as observed in the SQM calculation $[1,2]$. So thinking of the properties like its extension for a given baryon content, its strangeness content and its charge, the SQM and the SHM objects are rather identical.

Heavy ion collisions may constitute the only way to produce metastable strange matter. If a quark gluon plasma has formed, the proposed mechanism for the distillation of SQM [1] might as well enhance the rate of produced SHM.

[*] J. Schaffner, C. B. Dover, A. Gal, C. Greiner and H. Stöcker, Brookaven preprint

[1] C. Greiner, P. Koch and H. Stöcker, Phys. Rev. Lett. 58, 1825 (1987);

C. Greiner, D.H. Rischke, H. Stöcker and P. Koch, Phys. Rev. D 38, 2797 (1988);

C. Greiner and H. Stöcker, Phys. Rev. D 44, 3517 (1992).

[2] E. Witten, Phys. Rev. D30, 272 (1984); 


\section{Relativistic Heavy Ion Collisions}

\subsection{Medium modifications of parton cross sections}

(T. S. Biró, B. Müller, M. H. Thoma, X. N. Wang $\left[^{*}\right]$ )

The mechanisms that precede the formation of a thermalized, locally deconfined plasma of quarks and gluons in relativistic nuclear collisions have recently attracted considerable interest, because it was noticed that the preequilibrium phase may influence the yield of certain quark-gluon plasma signals, such as lepton pairs and hadrons containing heavy quarks. The space-time evolution of quark and gluon distributions has been investigated in the framework of the parton cascade model [1]. This model is based on the concept of the inside-outside cascade and evolves parton distributions by Monte-Carlo simulation of a relativistic transport equation involving lowest-order perturbative QCD scattering and parton fragmentation. Numerical studies $[2,3]$ have shown that phase-space equilibration of partons occurs over a period of $1-2 \mathrm{fm} / c$, and is initially dominated by gluon-induced processes.

The complexity of these calculations makes it difficult to obtain a lucid understanding of the dependence of the different time scales on various parameters and model assumptions. The motivation for our study was the desire to obtain a better physical understanding of the infrared cut-offs require $\mathrm{l}$ in the parton cascade model. The two cut-off parameters employed in ref. [1], the minimal transverse momentum transfer $p_{0}$ in binary parton interactions and the infrared cutoff $z_{\min }$ in parton fragmentation processes, were determined by comparison with cross-sections and particle multiplicities measured in nucleon-nucleon interactions at high energies. These values are assumed to reflect the transition between the perturbative and nonperturbative regimes of QCD in the normal vacuum. On the other hand, it is well known that color screening provides a natural cut-off of long-range interactions in a deconfined QCD plasma [1], and no artificial cut-off parameters are required to obtain finite perturbative cross sections in a dense medium.

A previous study [4] had shown that screening effects may be sufficiently strong immediately after the primary parton collision events in nuclear collisions at RHIC energies and beyond to provide the infrared cut-off required in the treatment of secondary parton interactions. Improved understanding of the suppression of fragmentation processes in a dense QCD plasma (Landau-Pomeranchuk effect) has also allowed to regard the infrared cut-off $z_{\min }$ as a medium-dependent effect in relativistic nuclear collisions (see Section 2.3 of this Report). We have shown how these medium effects can be utilized to obtain a parameter-free set of equations, based on perturbative QCD in a dense partonic medium, that describes the evolution of quark and gluon distributions towards equilibrium. Arbitrary cut-off parameters enter only into the description of the primary semi-hard parton scattering, where one must continue to rely on a comparison with nucleon scattering data. After this short initial phase, however, the approach towards an equilibrated quark-gluon plasma will be described without need for arbitrary parameters.

The kinematic separation of partons with different rapidity establishes conditions required for the validity of continuum dynamics after a short time of the order of 0.3 $\mathrm{fm} / c$, when the momentum space distribution of partons is roughly is otropic locally and approximately exponential. Since we are primarily interested in the chemical equilibration 
of the parton gas, we assume that the parton distributions can be approximated by thermal phase space distributions with non-equilibrium fugacities $\lambda_{i}$. Since we are interested in understanding the basic mechanisms underlying the formation of a chemically equilibrated quark-gluon plasma, and the essential time-scales, we restrict our considerations to the dominant reaction mechanisms for the equilibration of each parton flavour:

$$
g g \leftrightarrow g g g, \quad g g \leftrightarrow q \bar{q} .
$$

The cross sections $\sigma_{3}=\sigma(g g \rightarrow g g g)$ and $\sigma_{2}=\sigma(g g \rightarrow q \bar{q})$ contain infrared singularities if calculated in naive perturbation theory. These divergences are cured by the resummation of subsets of diagrams (hard thermal loops), which take screening effects into account. This method makes use of effective propagators and vertices, which show a complicated momentum dependence. In order to calculate $\sigma_{2}$ and $\sigma_{3}$ we adopt a simplified version of this idea by introducing momentum independent screening masses into the propagators, whenever infrared divergences arise otherwise. In the case of the stopping power and the viscosity this approximation provides quantitatively good results.

We write the triple differential radiative gluon-gluon cross section in the form

$$
\frac{d \sigma^{2 \rightarrow 3}}{d^{2} q_{\perp} d y d^{2} k_{\perp}} \approx C_{g g} \frac{2 \alpha_{s}^{2}}{\left(q_{\perp}^{2}+\mu_{D}^{2}\right)^{2}}\left[\frac{C_{A} \alpha_{s}}{\pi^{2}} \frac{q_{\perp}^{2}}{k_{\perp}^{2}\left(\mathbf{k}_{\perp}-\mathbf{q}_{\perp}\right)^{2}}\right]
$$

where $\mu$ is the Debye screening mass in the QCD plasma. $\mathbf{k}_{\perp}$ denotes the transverse momentum, $y$ the longitudinal rapidity of the radiated gluon, and $\mathbf{q}_{\perp}$ the momentum transfer in the elastic collision. In the presence of a dense medium the emission of radiation is suppressed, if the gluons scatter again before the emission is completed (Landau-Pomeranchuk effect), leading to the condition

$$
k_{\perp} \Lambda_{f}>2 \cosh y
$$

where $\Lambda_{f}$ is the mean free path of a gluon. The contribution from soft radiation is strongly suppressed by this effect. The integrated elastic gluon-gluon cross section in the medium

$$
\sigma^{2 \rightarrow 2}=2 \pi C_{g g} \frac{\alpha_{s}^{2}}{\mu_{D}^{2}},
$$

yields a fugacity independent mean free path

$$
\Lambda_{f}^{-1}=\sigma^{2 \rightarrow 2} n_{g}=\frac{9}{8} a_{1} \alpha_{s} T .
$$

To obtain the gluon production rate $R_{3}$ we integrate the differential cross section over momentum transfer $\mathbf{q}_{\perp}$ and the phase space of the radiated gluon. The collinear singularity in the differential cross section must be handled appropriately, but we have found that the results are not strongly dependent on the precise way of doing so. The chemical gluon equilibration rate $R_{3}=\frac{1}{2} n_{g} \sigma_{3}$ scales with the temperature linearly but is a complicated function of the gluon fugacity. The solid line in the Figure shows the scaled rate $R_{3} / T$ versus $\lambda_{g}$ for a coupling constant $\alpha_{s}=0.3$. The dotted line corresponds to the analytical fit

$$
R_{3}=2.1 \alpha_{s}^{2} T\left(2 \lambda_{g}-\lambda_{g}^{2}\right)^{1 / 2} . n
$$




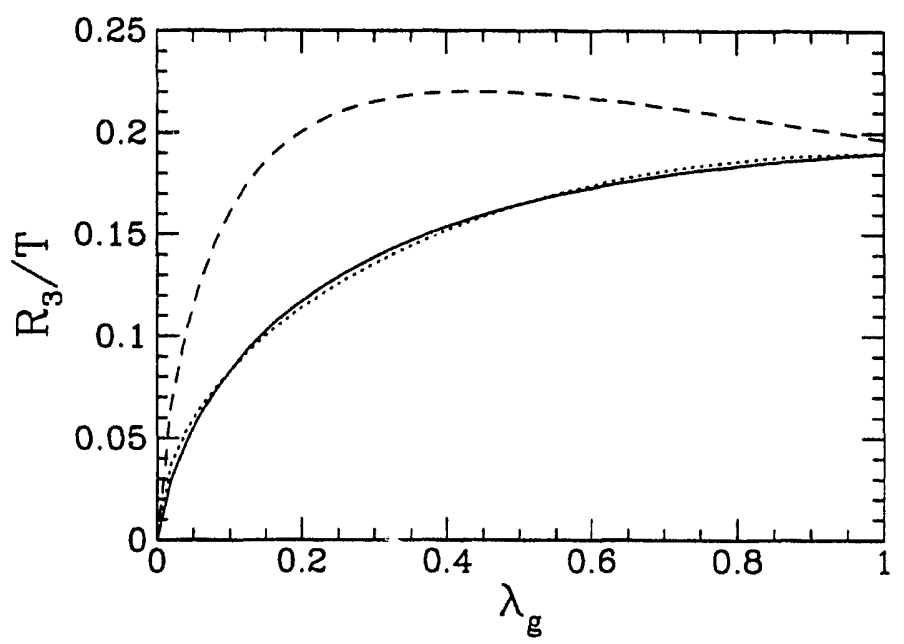

Fig. 1: Reduced gluon density equilibration rate $R_{3} / T$ as function of gluon fugacity.

The total cross section for the process $g g \rightarrow q \bar{q}$ is dominated by the Compton diagrams involving exchange of a virtual light quark in the $t$ - or $u$-channel. For massless quarks the differential cross section diverges as $u, t \rightarrow 0$, hence the medium-induced effective quark mass plays a crucial role. Since the divergence of the cross section is only logarithmic in this case, we obtain a sufficiently good estimate by simply substituting the effective thermal quark mass

$$
{ }^{2}=\left(\lambda_{g}+\frac{1}{2} \lambda_{q}\right) \frac{4 \pi}{9} \alpha_{s} T^{2}
$$

as cutoff in the divergent integral over momentum transfer. Integrating over thermal gluon distributions and inserting the average thermal collision energy $18 T^{2}$ in the logarithm, we find

$$
\sigma_{2} \approx N_{f}\left\langle\sigma_{q}\right\rangle \approx N_{f} \frac{\pi \alpha_{s}^{2}}{48 T^{2}}\left(\ln \frac{81}{2 \pi \alpha_{s} \lambda_{g}}-\frac{7}{4}\right)^{2} .
$$

For $\alpha_{s}=0.3$ in the logarithm and neglecting $\lambda_{q}$, the light quark production rate becomes

$$
R_{2}=\frac{1}{2} \sigma_{2} n_{g} \approx 0.064 N_{f} \alpha_{s}^{2} \lambda_{g} T\left(\ln \frac{7.5}{\lambda_{g}}\right)^{2} .
$$

[*] T. S. Biró, E. van Doorn, B. Müller, M. H. Thoma, and X. N. Wang, preprint DUKETH-93-46 (submitted to Phys. Rev. C).

[1] K. Geiger and B. Müller, Nucl. Phys. B369, 600 (1992).

[2] K. Geiger, Phys. Rev. D46, 4965 and 4986 (1992).

[3] E. Shuryak, Phys. Rev. Lett. 68, 3270 (1992).

[4] T. Biró, B. Müller, and X. N. Wang, Phys. Lett.B283, 171 (1992). 


\subsection{Parton Equilibration}

(T. S. Biró, E. van Doorn, B. Müller, M. H. Thoma, X. N. Wang $\left.{ }^{[*}\right]$ )

From numerical studies of parton cascades, as well as nd from more schematic considerations, a picture involving three distinct stages of parton evolution has emerged:

(1) Gluons "thermalize" very rapidly, reaching approximately isotropic momentum space distributions after a time of the order of $0.3 \mathrm{fm} / c$.

(2) Full equilibration of the gluon phase space density takes considerably longer.

(3) The evolution of quark distributions lags behind that of the gluons, because the relevant QCD cross sections are suppressed by a factor 2-3.

The complexity of these calculations makes it difficult to obtain a lucid understanding of the dependence of the different time scales on various parameters and model assumptions. In order to obtain this insight we reduced the parton cascade, using arguments of boost invariance and rapid thermalization of partons, to a set of rate equations describing the temporal evolution of the densities of gluons and quarks. The rates for the partochemical reactions $g g \leftrightarrow g g g, \quad g g \leftrightarrow q \bar{q}$ were calculated in thermal perturbation theory, taking into account medium corrections such as color screening and radiation suppression (see Section 2.1. of this Report).

Restricting to these reactions and assuming that elastic parton scattering is sufficiently rapid to maintain local thermal equilibrium, the evolution of the parton densities is governed by the masiter equations:

$$
\begin{aligned}
& \partial_{\mu}\left(n_{g} u^{\mu}\right)=n_{g}\left(R_{2 \rightarrow 3}-R_{3 \rightarrow 2}\right)-\left(n_{g} R_{g \rightarrow q}-n_{q} R_{q \rightarrow g}\right) \\
& \partial_{\mu}\left(n_{q} u^{\mu}\right)=\partial_{\mu}\left(n_{\bar{q}} u^{\mu}\right)=n_{g} R_{g \rightarrow q}-n_{q} R_{q \rightarrow g},
\end{aligned}
$$

where $R_{2 \rightarrow 3}$ and $R_{3 \rightarrow 2}$ denote the rates for the process $g g \rightarrow g g g$ and its reverse, and $R_{g \rightarrow q}$ and $R_{q \rightarrow g}$ those for the process $g g \rightarrow q \bar{q}$ and its reverse, respectively. The temperature evolves according to the hydrodynamic equation

$$
\partial_{\mu}\left(\epsilon u^{\mu}\right)+P \partial_{\mu} u^{\mu}=0,
$$

where viscosity effects have been neglected. In order to obtain simple solutions we will assume that the expansion of the parton fireball is purely longitudinal, yielding Bjorken's scaling solution of the hydrodynamic equation. This assumption is expected to be very well satisfied during the early expansion phase of the fireball, especially at proper time $\tau \ll R_{A}$, where $R_{A}$ is the transverse radius of the fireball. We express the densities in terms of temperature and fugacities according to the perturbative expressions

$$
n_{g}=\lambda_{g} \frac{16}{\pi^{2}} \zeta(3) T^{3}, \quad n_{q}=\lambda_{q} \frac{9}{2 \pi^{2}} \zeta(3) N_{f} T^{3} ;
$$

where $N_{f}$ denotes the number of active flavors, and the energy density

$$
\varepsilon=3 P=\left[8 \pi^{2} / 15 \lambda_{g}+7 \pi^{2} N_{f} / 40\left(\lambda_{q}+\lambda_{\bar{q}}\right)\right] T^{4} .
$$

We have solved the rate equations and the energy conservation equation simultaneously by numerical integration using a fourth order Runge-Kutta method. The initial 
conditions for these rate equations are the number density $n_{0}=n\left(t_{\text {iso }}\right)$ and the transverse energy density $\varepsilon_{T}=\varepsilon_{T}\left(t_{\text {iso }}\right)$ of gluonic partons were determined from a simulation of perturbative QCD cross sections for the primary partons contained in the colliding nuclei in the HIJING code. We obtain $n_{0}=2.4 \mathrm{fm}^{-3}$ and $\varepsilon_{0}=3.5 \mathrm{GeV} / \mathrm{fm}^{3}$ at RHIC energy, and $n_{0}=23 \mathrm{fm}^{-3}$ and $\varepsilon_{0}=52 \mathrm{GeV} / \mathrm{fm}^{3}$ at LHC energy. Quarks contribute a smaller amount to the initial parton energy density, because the quark-producing cross sections are smaller in perturbative QCD than those for gluon production. The total quark contribution to the energy density we estimate as 30 percent, yielding total initial energy densities of 5 $\mathrm{GeV} / \mathrm{fm}^{3}$ at RHIC and $70 \mathrm{GeV} / \mathrm{fm}^{3}$ at LHC.

The evolution of temperature and the fugacities are shown in Figure 1. We find that the parton gas cools considerably faster than predicted by Bjorken's scaling solution $\left(T^{3} \tau=\right.$ const.), because the production of additional partons aproaching the chemical equilibrium state consumes an appreciable amount of energy. The accelerated cooling on the other hand impedes the chemical equilibration process, which is more apparent at RHIC (Fig. 1a) than at LHC energies (Fig. 1b).
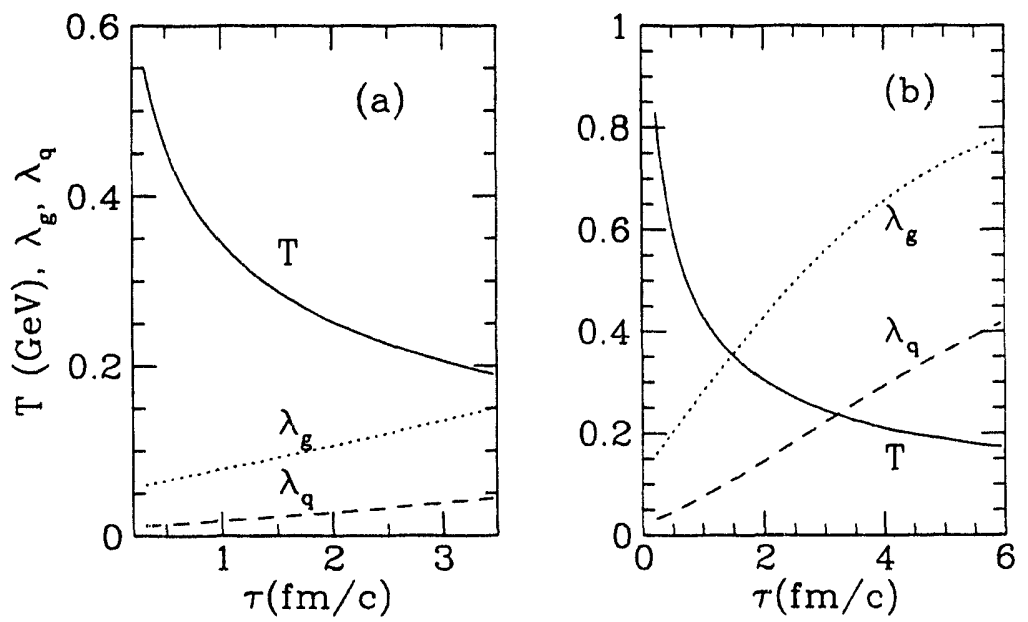

Fig. 1: Gluon and quark density equilibration at (a) RHIC and (b) LHC energies for $\mathrm{Au}+\mathrm{Au}$ collisions.

In order to see where the perturbative description of the parton plasma is applicable we investigate the time evolution of the total energy density. The solid lines in Fig. 2 correspond to the initial conditions mentioned above, whereas dashed line corresponds to a more optimistic estimate of the initial gluon production at RHIC $\left(\lambda_{g} \approx 0.18\right)$. One realizes that the perturbative parton plasma has a lifetime of $1-2 \mathrm{fm} / c$ at RHIC, while at LHC the plasma may exist in a deconfined phase as long as $5-6 \mathrm{fm} / c$. A higher initial value of $\lambda_{g}$ can result from rescattering of gluons during the "free-streaming" period after the end of initial semihard scattering. Microscopic parton cascade simulations indicate a rapid increase of gluon density during this pre-thermal phase.

From our investigation emerges the following scenario of a nuclear collision at collider energies: Within $0.2-0.3 \mathrm{fm} / c$ a dense parton gas is produced at central rapidities, which can be described as a locally thermalized, but not chemically equilibrated quark- 
gluon pasma. In the fragmentation region, where the parton gas created by minijets is not sufficiently dense to screen color fields, strings and color ropes are formed [1], which decay by non-perturbative QCD processes on a time scale of about $1 \mathrm{fm} / c$. These strings extend between the wounded nuclei and the surface of the parton plasma, penetrating up to a screening length determined by the actual Debye mass. The main difference to the conventional Bjorken scenario [2] is the far shorter formation time in the central rapidity region, corresponding to a much higher initial temperature.

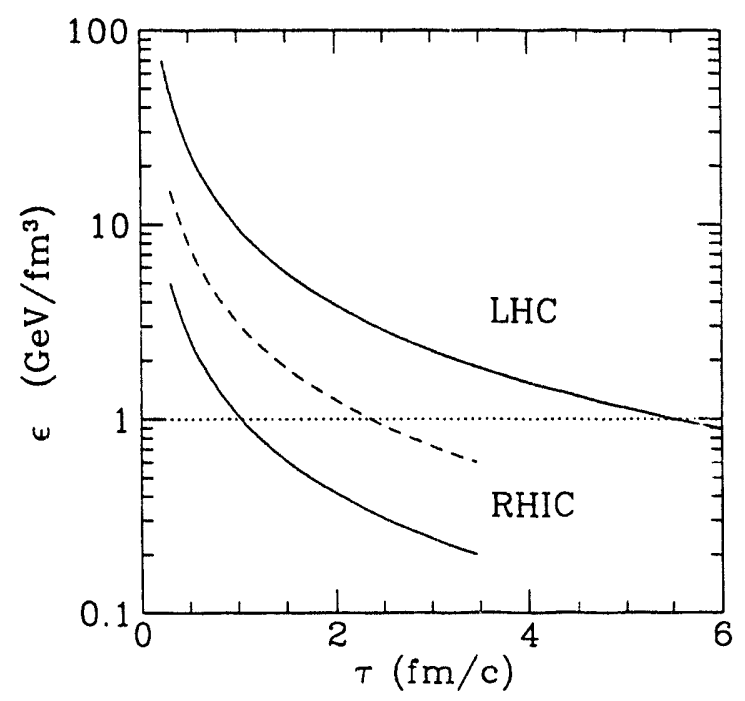

Fig. 2: Evolution of the energy density of the parton plasma in $\mathrm{Au}+\mathrm{Au}$ collisions.

Our results support the picture that the highly excited parton plasma created in ultrarelativistic nuclear collisions is initially mainly a gluon plasma. This has important implications for several experimental signals associated with quark-gluon plasma formation: Rapid gluon thermalization at a high initial temperature leads to a substantial thermal contribution to the total yield of charmed quarks. The lack of chemical equilibration in the quark and gluon densities, on the other hand, causes a severe depletion of the number of emitted lepton-pairs compared with the naive thermal estimate. However, the shift towards higher invariant masses of the steeply falling lepton-pair spectrum due to the larger initial temperature in the plasma can conceivably offset this suppression.

[*] T. S. Biró, E. van Doorn, B. Müller, M. H. Thoma, and X. N. Wang, preprint DUKETH-93-46 (submitted to Phys. Rev. C).

[1] T. Biró, H. B. Nielsen, and J. Knoll, Nucl. Phys. B245, 449 (1984);

K. J. Eskola and M. Gyulassy, preprint LBL-33150.

[2] J. D. Bjorken, Phys. Rev. D27, 140 (1983). 


\subsection{Multiple Collisions and Induced Gluon Bremsstrahlung in QCD (M. Gyulassy, X.-N Wang)}

Radiative energy loss of an ultra-relativistic particle passing through dense matter is of interest not only because of its many practicle applications, but also because it illustrates a novel type of destructive interference phenomena related to the finite formation time,

$$
\tau(k) \sim \hbar / \Delta E(k) \sim 2 \omega / k_{\perp}^{2} \sim 2 / \omega \theta^{2},
$$

of quanta with large four momentum, $k^{\mu}=\left(\omega, k_{z}, \mathbf{k}_{\perp}\right)$, at small angles, $\theta \approx k_{\perp} / \omega$. In effect, $\tau(k)$, is the minimal time needed in the laboratory to resolve the transverse wavepacket of the quanta with, $\Delta x_{\perp} \sim \hbar / k_{\perp}$, from the wavepacket of its high energy $\left(E_{0} \gg \omega\right)$ parent. Destructive interference between radiation amplitudes associated with multiple collisions can be expected when the mean free $\lambda$ is short compared to the formation time. When $\tau(k) \gg \lambda$, the emitted quanta cannot resolve different elastic scattering centers and the assumption of independent contributions from each seperate scattering in the medium breaks down. This effect, first studied in QED and then in other field theories, is often referred to as the Landau-Pomeronchuck-Migdal (LPM) effect.

We try to study the same effect in QCD within multiple scattering formalism. We found that the gluon spectrum induced from $m$ number of scatterings can be written in the form,

$$
\frac{d^{3} n_{m}}{d^{3} k} \equiv C_{m}(k) \frac{d^{3} n_{1}}{d^{3} k} .
$$

where,

$$
\omega \frac{d^{3} n_{1}}{d^{3} k}=\left\langle\frac{\alpha C_{A}}{\pi^{2}} \frac{q_{\perp}^{2}}{k_{\perp}^{2}\left(\mathbf{k}_{\perp}-\mathbf{q}_{\perp}\right)^{2}}\right\rangle
$$

is the radiation distribution from a single isolated collision, and the color formation factor $C_{m}(k)$ is defined by

$$
C_{m}(k)=\frac{1}{C_{2}^{m} C_{A} d} \sum_{i=1}^{m}\left(C_{i i}+2 R e \sum_{j=1}^{i-1} C_{i j} e^{i\left(z_{i}-z_{j}\right) / \tau(k)} F_{i j}(k)\right),
$$

in terms of color coefficients,

$$
C_{i j}=\operatorname{Tr}\left(a_{m} \cdots\left[c, a_{i}\right] \cdots a_{1} a_{1} \cdots\left[a_{j}, c\right] \cdots a_{m}\right),
$$

and current correlation functions

$$
F_{i j}(k)=\left\langle\vec{\jmath}_{i} \cdot \vec{\jmath}_{j}\right\rangle /\left\langle\left|\vec{\jmath}_{i}\right|^{2}\right\rangle,
$$

with $\vec{\jmath}_{i}$ given by

$$
\overrightarrow{\jmath_{i}}(k)=2 i g\left(\frac{\mathbf{k}_{\perp}}{k_{\perp}^{2}}+\frac{\mathbf{q}_{\perp i}-\mathbf{k}_{\perp}}{\left|\mathbf{q}_{\perp i}-\mathbf{k}_{\perp}\right|^{2}}\right)
$$


In Eq. $4,5, a_{i}$ are the color matrices of $\mathrm{SU}(\mathrm{N})$ in $d$ dimension representation. $C_{2}$ and $C_{A}$ are defined as $a a=C_{2} 1_{d}$ and $[a, b] a=-\frac{C_{A}}{2} b$.

In the soft radiation limit, we can calculate the color formation factor and thus obtain the radiative energy loss as

$$
d E / d x \sim \frac{1}{2} \alpha C_{2} \mu^{2} \log \left(s / 4 \mu^{2}\right),
$$

where $\mu \sim g T$ and $s \sim 6 E_{0} T$ for a parton propagating through a QGP at temperature $T$. Thus unlike in a single scattering, where the radiation intensity is proportional to $C_{A}$ via Eq.(3), the extra induced radiation per mean free path is proportional to the $C_{2}$ of the radiating parton. This means that gluons radiate $C_{A} / C_{F}=9 / 4$ more gluons than quarks for $S U(3)$.

\subsection{Branching Processes and the Multiple Production}

(S. G. Matinyan $[*]$ )

The evident violation of KNO scaling at the energy of the CERN $\bar{p} p$ collider attract much attention to the Negative Binomial Distribution (NBD) which describes fairly well the overall features of the multiplicity distributions in different processes, in different rapidity ranges and over a wide energy interval.

We have used the general theory of the branching process for establishing the relation

$$
k=\frac{a \bar{n}}{\bar{n}-b}, \quad a=3.06 \pm 0.06 ; b=6.95 \pm 0.08
$$

gives the possibility to describe the overall data on the multiplicity distribution in $\vec{p} p$ collisions for energies ur to $900 \mathrm{GeV}$ and to make several predictions for higher energies.

These predictions are i) the restoration of KNO-like scaling in the multi $\mathrm{TeV}$ region, ii) asymptotically constant values for $Q_{q}$-moments $\left(C_{q} \equiv\left\langle n^{q}\right\rangle /\langle n\rangle^{q}\right)$ depending on $q$, iii) the validity of Wroblewski's rule for high energies, iv) the asymptotic value of a peak for the KNO distribution

$$
\psi(z) \equiv \bar{n} P_{n}(z) \quad(z=n /\langle n\rangle) .
$$

This general approach is free from controversies of NBD associated with the extrapolation of the parameter $k$ to unity. The model is compared with other models using the detailed branching processes of quarks and gluons.

[*] S. G. Matinyan and E. B. Prokhorenko, preprint DUKE-TH-93-51. 


\section{Finite-Temperature QCD}

\subsection{Magnetic Screening in Thermal Yang-Mills Theories}

(T.S. Biró, B. Müller [*])

The screening of static magnetic gauge fields in Yang-Mills theories at high temperature has long proved to be intractable by analytic techniques. There exist well known arguments that the inverse screening length $\mu_{M}$ must be of order $g^{2} T$, and this has been partially supported by numerical calculations in the framework of lattice gauge theory [1] which have yielded the result $\mu_{M}=(0.27 \pm 0.03) g^{2} T$ for SU(2). The recently developed resummation techniques for the finite-temperature gauge theories cure many infrared divergences arising in the perturbative expansion, but not those associated with long-range static magnetic gauge fields. Although the Schwinger-Dyson equation that determines $\mu_{M}$ has been identified [2], its solution remains unknown.

We propose a new approach to the calculation of $\mu_{M}$, which is not based on a perturbative expansion in the gauge coupling constant $g$. Our starting point is the observation that the combination $g^{2} T$ defines an inverse length without any factor involving powers of Planck's constant $\hbar$. One may therefore speculate that $\mu_{M}$ can be calculated from classical Yang-Mills statistical mechanics, with quantum effects providing corrections of higher order in $g$.

In order to obtain the magnetic screening length one usually investigates the linear response of the medium to an infinitesimal external magnetic field. The magnetic field, which couples to the monopole charge, is curl-free and can be expressed by a scalar magnetic potential, $B_{i}^{a}=-\partial_{i} \phi^{a}$. The application of such an external magnetic field modifies the monopole charge density in the plasma, so the partition function becomes

$$
Z(\phi)=\operatorname{Tr} e^{-\beta\left(\hat{H}-\phi^{a} \hat{Q}^{a}\right)},
$$

where $\hat{Q}^{a}$ is the magnetic charge operator and the trace "Tr" runs over all possible states of the Yang-Mills field. Let us denote a given color charge state by $\left|q, q_{3}\right\rangle$, where $q$, the eigenvalue of the Casimir operator, is a multiplet index. A magnetic monopole state corresponds to the eigenvalue $q=1$. Taking into account the bosonic nature of excitations of the Yang-Mills field, the magnetic monopole partition function in the dilute gas limit becomes in the case of $\mathrm{SU}(2)$ :

$$
Z=\prod_{\omega} \prod_{q_{3}} \sum_{n=0}^{\infty} e^{-n\left(\beta \omega-q_{3} \Theta\right)},
$$

where $q_{3}$ runs over the possibilities $-1,0$ and +1 , and $\omega$ denotes the energy eigenvalues of the Hamiltonian. From this partition function we obtain the magnetic color charge density, which in the weak external field limit $(\Theta \ll 1)$ becomes:

$$
\rho^{a}=\frac{1}{\beta V} \frac{\partial}{\partial \phi^{a}} \ln Z=-\frac{N}{V g^{2} T} Z_{M} \phi^{a} .
$$


Since the magnetic monopole charge density is the source of the divergence of the magnetic field, we arrive at an equation describing the linear polarizability of a magnetic monopole gas, which yields the effective magnetic screening mass

$$
\mu_{M}=\left(\frac{N Z_{M}}{\left(g^{2} T\right)^{3} V}\right)^{\frac{1}{2}} g^{2} T
$$

In order to calculate the nontrivial factor we need to obtain the partition function $Z_{M}$. Although simple scaling arguments show that there may be a characteristic monopole size $R_{0}$ contributing dominantly to the partition sum $Z_{M} \sim \int \frac{d R}{R^{5}} \exp \left(-R_{0} / R\right)$, no classical monopole solution exists, which would offer a stable stationary point of the path integral defining the partition function. The lack of a basis for a semiclassical analysis of the statistical mechanics of monopole solutions in non-abelian gauge theories apparently vitiates an analytical approach. We have found a simple, yet elegant way how to circumvent the stability problem.

$\mathrm{SU}(2)$ gauge field configurations which carry one unit of magnetic charge must asymptotically look like a monopole $(j=0)$ mode of the operator $\hat{J}=\hat{S}+\hat{I}+\hat{L}$, where $\hat{S}$ and $\hat{I}$ denote the generators of spin and color spin in the adjoint representation and $\hat{L}$ is the generator of orbital angular momentum for the gauge field. Since the gauge field belongs to the representation $S=I=1$, there are three different possibilities to construct a $j=0$ mode; namely $|L, T\rangle=|1,1\rangle,|0,0\rangle$, and $|2,2\rangle$ combinations, where the grand-spin quantum number $T$ is obtained from the eigenvalue of the Casimir operator $\hat{T}^{2}=T(T+1)$ with $\hat{T}=\hat{S}+\hat{I}$. The Wu-Yang monopole, known to be unstable, belongs to the $j=0$ mode of type $|L, T\rangle=|1,1\rangle$.

The most general ansatz for the monopole vector potential has the form

$$
A_{i a} \equiv \sum_{\alpha= \pm, 0} P_{i a}^{(\alpha)} A^{(\alpha)}=\frac{1}{r}\left(P_{i a}^{+}\left(u e^{i \phi}-i\right)+P_{i a}^{-}\left(u e^{-i \phi}+i\right)+P_{i a}^{0} w\right),
$$

where $u(r), \phi(r)$ and $w(r)$ are real functions of the radial variable only and we use the projectors $P_{i a}^{ \pm}=\frac{1}{2}\left(\delta_{i a}-n_{i} n_{a} \pm i \epsilon_{i a j} n_{j}\right), P_{i a}^{0}=n_{i} n_{a}$ with the unit radial vector $n_{i}=x_{i} / r$. The magnetic monopole charge seen from outside a sphere of radius $r$ can be obtained from the magnetic analogue of Gauss' law

$$
Q_{a}(r)=\frac{1}{4 \pi} \int d^{3} r \partial_{i} B_{i a}=\frac{1}{4 \pi} \oint_{r} n_{i} B_{i a}=n_{a}\left(1-u^{2}(r)\right) .
$$

This result shows that a monopole field configuration requires asymptotically $u \rightarrow 0$ as $r \rightarrow \infty$, irrespective to the fields $w(r)$ and $\phi(r)$. Introducing the scaled parameter $\tilde{\beta}=\frac{4 \pi}{g^{2} T}$, we find for the energy of this static configuration in the high- $T$ limit:

$$
\begin{aligned}
\beta E & =\tilde{\beta} \int_{0}^{\infty} d r\left[\left(\frac{d u}{d r}\right)^{2}+u^{2}\left(\frac{d \phi}{d r}+\frac{1}{r} w\right)^{2}+\frac{\left(1-u^{2}\right)^{2}}{2 r^{2}}\right] \\
& =\tilde{\beta} E[u]+\tilde{\beta} \int_{0}^{\infty} d r u^{2}\left(\frac{d \phi}{d r}+\frac{w}{r}\right)^{2}
\end{aligned}
$$


where one observes that only the field $u(r)$ is really a dynamical degree of freedom while $w(r)$ is non-dynamical and $\phi(r)$ is a cyclic variable. Closer inspection shows that the latter two really correspond to residual gauge degrees of freedom, and after some algebra the partition function reduces to a functional integral over $u(r)$ of the form:

$$
Z_{M}=\int \mathcal{D} u e^{-\tilde{\beta} E[u]} Z_{\mathrm{tr}}[u] Z_{\mathrm{rot}}[u] \prod_{j>0}^{\prime} Z_{j}[u],
$$

where the prime indicates that the zero-modes have been separated from the field modes with non-vanishing angular momentum $j$. We neglect these higher modes in the following.

Unfortunately, $E[u]$ does not have a stable saddle point $u_{0}(r)$. This lack of a basis for the semiclassical expansion, however, it does not preclude the calculation of the partition function for gauge field configurations $u$ with monopole symmetry. It only implies that the evaluation of the functional integral cannot be restricted to integration over Gaussian fluctuations around a classical solution $u_{0}(r)$. Our new method for evaluating this functional integral, where $u(r)$ satisfies the boundary conditions $u(\infty)=0, u(0)=1$, involves adding a stabilizing term to the expression for $E[u]$,

$$
E[u]+\lambda^{2} D[u]=\int_{0}^{\infty} d r\left[\left(\frac{d u}{d r}\right)^{2}+\frac{\left(1-u^{2}\right)^{2}}{2 r^{2}}\right]+\frac{1}{2} \lambda^{2} \int_{0}^{\infty} d r\left(1-\left(1-u^{2}\right)^{2}\right) .
$$

This introduces a length scale that favors monopole configurations of a particular core size. We then integrate over the dummy scale in such a way that the partition function remains unchanged:

$$
Z_{M}=\int_{0}^{\infty} d \lambda 2 \tilde{\beta} \int \mathcal{D} u D[u] e^{-\tilde{\beta}\left(E[u]+\lambda^{2} D[u]\right)} Z_{\mathrm{tr}} Z_{\mathrm{rot}} .
$$

The functional integral over $u$ can now be approximated by a Gaussian integration around the lowest energy stationary solution $u_{0}(r)$ of the exponent. Since there is no other scale involved besides the dummy parameter $\lambda$, the solution is solely a function of the dimensionless variable $x=\lambda r$, which satisfies the equation

$$
\frac{d^{2} u_{0}}{d x^{2}}+u_{0}\left(1-u_{0}^{2}\right)\left(\frac{1}{x^{2}}-1\right)=0 .
$$

The function $u_{0}(r)$, obtained by numerical integration, is displayed in the Figure, together with the monopole charge contained inside the radius $r$. The ground state energy scales as $\lambda a$, whereas $D[u]$ scales as $b / \lambda$. We find $a=1.469, b=0.695$.

The zero-mode factors are found to be:

$$
Z_{\mathrm{tr}}=\left(\frac{2}{3} a\right)^{\frac{3}{2}} \lambda^{\frac{9}{2}} V, \quad Z_{\mathrm{rot}}=\left(\frac{2}{5} a\right)^{\frac{3}{2}} \lambda^{-\frac{3}{2}} 8 \pi^{2} ;
$$

their product scales like $\lambda^{3}$. The integral over $\lambda$ can now be carried out, and we finally obtain the monopole density in the classical limit as

$$
\rho_{M}=\frac{1}{V} Z_{M}=\frac{12 b}{15^{3 / 2} \pi a}\left(g^{2} T\right)^{3} .
$$




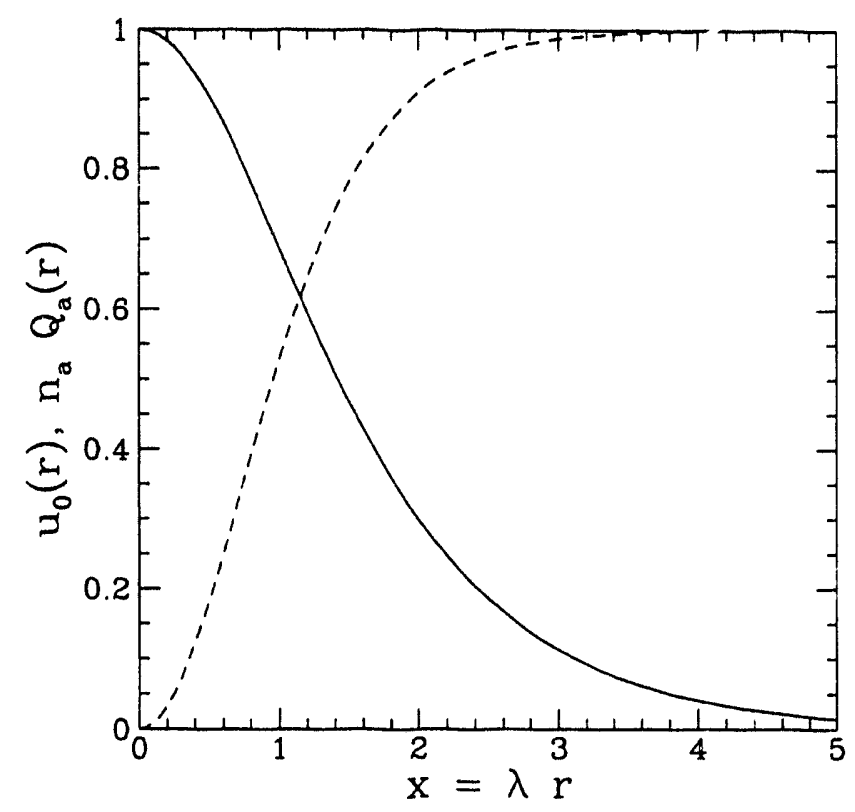

Fig.: The classical magnetic monopole soliton $u_{0}(r)$ which minimizes the energy functional $E[u]+\lambda^{2} D[u]$ is shown as solid line. The resulting monopole charge contained inside a sphere of radius $r$ is represented by the dashed line.

We finally evaluate the scaled determinantal contribution due to the spherical fluctuations around the soliton-monopole solution $u_{0}(r)$. It turns out that this determinant has a value very close to unity. Altogether we find the following result for the magnetic screening mass in $\mathrm{SU}(2)$ :

$$
\mu_{M} \approx 0.255\left(g^{2} T\right),
$$

which is amazingly close to the value obtained in lattice simulations. Of course, the ontributions due to higher multipole modes, which were neglected, may change this result quantitatively. However, the fact that the determinantal factor for radial fluctuations is very close to unity gives hope that the influence of those higher modes will be small.

[*] T. S. Biró and B. Müller, preprint DUKE-TH-92-42, Nucl. Phys. A (in press).

[1] A. Billoire, G. Lazarides, and Q. Shafi, Phys. Lett. 103B, 450 (1981);

T. A. DeGrand and D. Toussaint, Phys. Rev. D25, 526 (1982).

[2] O. K. Kalashnikov, Phys. Lett. B279, 367 (1992).

\subsection{Magnetic Field Correlation on the Lattice}

(C. Gong, B. Müller)

In the finite temperature gauge field theory, one of the outstanding problems is the infrared divergence due to the lack of a screening on the magnetic part of the interaction. 
It is argued that the magnetic screening mass $m_{m}$ shall be of the order of $g^{2} T$ [1] but it is still not clear how it comes about in the formalism of the finite temperature perturbative calculations. On the other hand, lattice calculations have been carried out and the above speculation of $g^{2}$ is confirmed [2], but the uncertainty is large. So further study is necessary. We want to address this problem in the classical dynamical context. The motivation is that if $m_{m}$ is of the order of $g^{2} T$, then it shall appear as a classical quantity, just as the damping rate we have studied previously (see section 4.2.).

The most direct method to study the screening of the magnetic interaction is to study the spatial correlation function of magnetic fields, $C(r) \equiv\langle B(r) B(0)\rangle$. The complexity in the non-abelian theory is that the field variables are gauge covariant, but not gauge invariant. To have a gauge invariant correlator, we need to insert connections. On the lattice, a connected correlator is just a closed spatial Wilson loop $W(r)$ depicted in Fig.1.

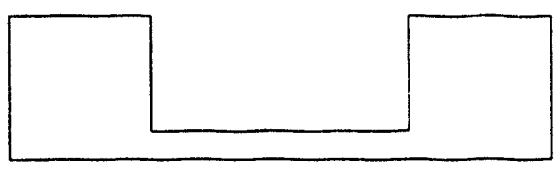

Fig. 1: A spatial Wilson loop corresponding to the magnetic field correlation.

At the two ends of the loop are two plaquettes, which correspond to $B(x)$ and $B(0)$. The two plaquettes are connected by a neck. In the limit of small $B(r)$, this Wilson loop related to the previous correlator as $W(r) \sim 2-E_{m}-1 / 2 C(r)$, where $E_{m}$ is the average magnetic energy per plaquette. If we observe the exponential decay of $C(r)$ with distance $r$, then we can identify the decay rate as the magnetic screening mass.

We have numerically studied $C(r)$ at different energies for $\mathrm{SU}(2)$ and also studied $\mathrm{U}(1)$ for comparison. In all cases, $C(r)$ decays rapidly and there is almost no correlation at all among the neighbouring plaquettes. This has to do with the fact that the correlation we define is the dipole-dipole correlation which decays as $r^{-4}$ even if without magnetic screening. So it seems difficult to extract magnetic srceening from the study of magnetic field correlation.

[1] A. D. Linde, Phys. Lett. 96 B, 289 (1980); D. J. Gross, R. D. Pisarski, and L. G. Yaffe, Rev. Mod. Phys. 53, 43 (1981).

[2] A. Billoire, G. Lazarides, and Q. Shafi, Phys. Lett. 103B, 450 (1981); T. A. DeGrand and D. Toussaint, Phys. Rev. D25, 526 (1982).

\subsection{The $\lambda \phi^{4}$ Theory on a Four-dimensional Torus} (C. Villarreal $[*]$ )

An ideal system for studying the response of the vacuum to interactions, thermal excitations, and boundary conditions is the $\lambda \phi^{4}$ theory constrained to a torus in $(3+1)$ dimensional space-time:

$$
\mathcal{L}=\left(\partial_{\mu} \phi^{*}\right)\left(\partial^{\mu} \phi\right)+m_{0}^{2} \phi^{*} \phi-\lambda\left(\phi^{*} \phi\right)^{2},
$$


where $m_{0}^{2}=-m^{2}$ is a negative squared mass. In particular, we are interested the possibility of developing self-sustained equilibrium configurations by a proper balance of the Casimir forces and the self-interaction. In order to study the energy density as function of the geometry, the torus has different spatial extension in every direction.

Following the usual discussion of symmetry breaking, the field is explicitly separated into a (real) classical vacuum expectation value $v$ plus quantum excitations: $\phi=v+\chi$, where $\chi=\chi_{1}+i \chi_{2}$, has vanishing vacuum and thermal expectation values. At the minimum of the classical potential given by $v_{0}^{2}=m_{0}^{2} / 2 \lambda$, the mean field masses show the values $m_{1}^{2}=m_{0}^{2} / 2 \lambda$ and $m_{2}=0$, i.e., $\chi_{2}$ is a Goldstone boson.

The constraint imposed by the toroidal geometry is implemented by requiring the fields to satisfy periodic boundary conditions in the spatial directions. the behavior of the system both at zero and finite temperature may be investigated by means of a standard method employed in finite-temperature field theories, i.e., to perform an analytical continuation into imaginary time. Thus, we consider quantum fields defined over the 4-torus with spatial periods $a, b, c$, and time period $i \beta$. The thermal free energy density $F_{\beta}=-(T / V) \ln Z_{\beta}$ is

$$
F_{\beta}=\frac{T}{V} \sum_{n_{1}, n_{2}, n_{3}}\left\{\ln \left[1-\exp \left(-\beta \omega_{1}\left(\mathbf{k}_{\mathbf{n}}\right)\right)\right]+\ln \left[1-\exp \left(-\beta \omega_{2}\left(\mathbf{k}_{\mathbf{n}}\right)\right)\right]\right\} .
$$

The finite-temperature contributions

$$
\begin{aligned}
F_{0}= & -\frac{m_{1}^{2}}{4 \pi^{2}} \sum_{n_{1}, n_{2}, n_{3}}^{*} \frac{K_{2}\left(m_{1} \sqrt{n_{1}^{2} a^{2}+n_{2}^{2} b^{2}+n_{3}^{2} c^{2}}\right)}{n_{1}^{2} a^{2}+n_{2}^{2} b^{2}+n_{3}^{2} c^{2}} \\
& -\frac{m_{2}^{2}}{4 \pi^{2}} \sum_{n_{1}, n_{2}, n_{3}}^{*} \frac{K_{2}\left(m_{2} \sqrt{n_{1}^{2} a^{2}+n_{2}^{2} b^{2}+n_{3}^{2} c^{2}}\right)}{n_{1}^{2} a^{2}+n_{2}^{2} b^{2}+n_{3}^{2} c^{2}}
\end{aligned}
$$

can be evaluated by a straightforward numerical calculation. This expression coincides with previous results for the total Casimir energy density [1]. On the other hand, the terms associated to the zero-point field must be renormalized in order to get a finite result [2]. The renormalized effective potential is

$$
\begin{aligned}
U_{R}= & -m_{0}^{2}\left(1-\frac{\lambda}{8 \pi^{2}}\right) v^{2}+\lambda\left(1-\frac{5 \lambda}{16 \pi^{2}}\right) v^{4} \\
& +\frac{m_{1}^{4}}{64 \pi^{2}} \ln \left(\frac{m_{1}^{2}}{2 m_{0}^{2}}\right)+\frac{m_{2}^{4}}{64 \pi^{2}} \ln \left(\frac{m_{2}^{2}}{2 m_{0}^{2}}\right) .
\end{aligned}
$$

The self-interaction of the scalar field is taken into account in perturbation theory, where the propagators satisfy the torus boundary conditions. The final expression for finite two-loop contributions to the free energy is

$$
\begin{aligned}
F_{\text {loop }}= & \frac{3 \lambda T}{4 V}\left[\left(\Delta_{1}^{0}+\Delta_{1}^{\beta}\right)^{2}+\left(\Delta_{2}^{0}+\Delta_{2}^{\beta}\right)^{2}\right. \\
& \left.+\frac{2}{3}\left(\Delta_{1}^{0}+\Delta_{1}^{\beta}\right)\left(\Delta_{2}^{0}+\Delta_{2}^{\beta}\right)\right] .
\end{aligned}
$$


The total free energy density is $F=F_{0}+F_{\beta}+F_{\text {Loop }}+U_{R}$.

A variational calculation of the free energy density has been performed in the parameter space of the system, determined by the set $\left\{a, b, c, \lambda, m_{0}\right\}$. It is convenient to express every quantity in units of $m_{0}$. Therefore we have defined adimensional variables $\hat{a_{i}} \equiv a_{i} m_{0}$ ( $a_{i}$ is any spatial length), $\hat{M}_{i} \equiv M_{i} / m_{0}, \hat{T} \equiv T / m_{0}$, and $\hat{v}=v / m_{0}$. In order to simplify matters we considered that $a=b$, and defined the ratio $R \equiv a / c$. When one spatial dimension is strongly compactified, $R \gg 1$, and the quantization volume is constrained to a thin wall in space. For $R \ll 1$, the quantization volume becomes a string configuration. The free energy surface in a unit volume for a given value of $\lambda$ shows a saddle behavior, with the parameters corresponding to the cubical configuration defining the "top" of the saddle. The string and the wall configurations lower the free energy density, and destabilize the system. This occurs whenever the coupling constant is smaller than a certain $\lambda_{c}$. However, as $\lambda$ reaches the critical value $\lambda_{c}=0.4$, the free energy surface suffers an abrupt modification, and the string configuration becomes stable.

A transverse section of the free energy surface at the critical point reveals that the minimum of the free energy density for the cube is only slightly shifted with respect to the mean field value $\hat{v}=1.12$. In fact, the isotropic mean field result is recovered as soon as $\hat{a_{i}} \approx 5$ in every spatial direction. On the other hand, when the system is described by the string configuration the minimum of the effective potential is located at $\hat{v}_{c}=5.65$. At this point $R_{c}=.002$ and, taking into account that the volume is fixed, $\hat{a}_{c}=\hat{b}_{c}=0.125$. Apparently, $\mathrm{nc}$ reasonable value of $\lambda$ can render a stable wall configuration.

[*] C. Villarreal, preprint DUKE-TH-92-45.

[1] J. Ambjorn and S. Wolfram, Ann. Phys. 147, 1 (1983).

[2] J. I. Kapusta, Finite-Temperature Field Theory (Cambridge University Press, Cambridge, 1988). 


\section{Real Time Lattice Gauge Theory}

\subsection{Lyapunov Exponent of SU(3) Gauge Theory}

(C. Gong [*])

In this investigation we use a classical lattice gauge method [1] to study chaoticity in $\mathrm{SU}(3)$ lattice gauge theory. This is related to the entropy production and thermalization process in a highly excited gluon system, which is relevant in the study of the early universe and of relativistic heavy ion collisons, where the phase transition of nuclear matter to quark-gluon plasma is expected to occur. The proposed signatures of this transition depend critically on the time scale of the pre-equilibrium thermalization process. Studies show that the time scale of this process is short and the gluon sector thermalizes much faster compared to the quark sector $\left(\tau_{q}=3 \tau_{g}\right)$. This later fact makes it plausible to consider the thermalization of the gluon sub-system separately. But up to now, this process is still not completely understood because a fully non-perturbative quantum mechnical calculation is beyond our ability. On the other hand, our method not only leads to an exact and non-perturbative solution to this problem (in the classical limit), but also helps to explain the reason of a fast thermalization process in the classical language. That is the chaotic nature of the non-abelian gauge theory. We hope that our results will be helpful to a full quantum treatment in the future.

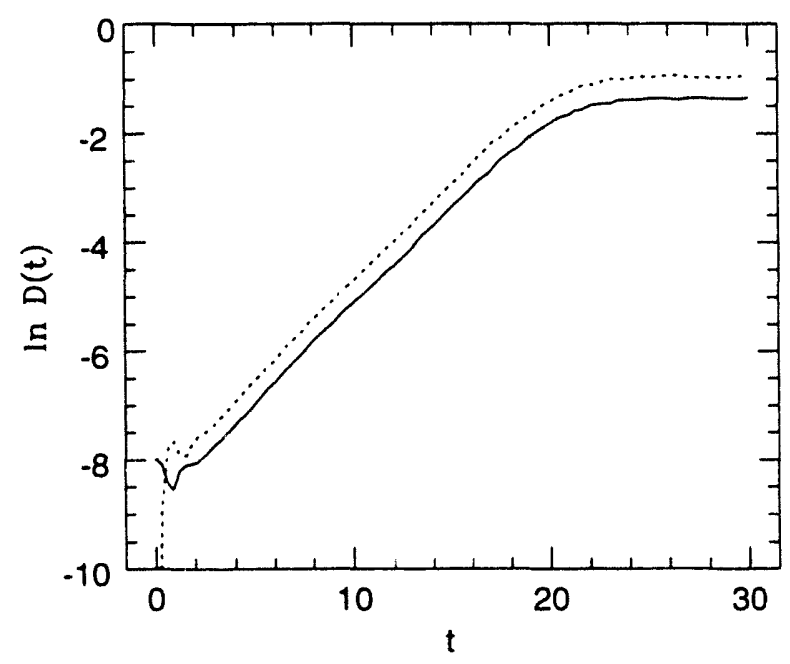

Fig. 1: Divergence of two classical trajectories in two different measures $D(t)$. The solid line corresponds to a definition of distance that is the summation of absolute value of local magnetic energy difference, the dotted line corresponds to that of electric energy. The latter is slightly vertically shifted.

Our study is based on the lattice Hamiltonian [2] for SU(3) gauge fields from which we can derive the classical equations of motion for the color electric and magnetic field components defined on the links of a three dimensional lattice. With these equations we can trace out the real time evolution of a given initial field configuration. 
Initially we put all color electric field components to be zero to avoid the presence of local static color charges on the lattice. Then we use a heat bath algorithm [3] to thermalize the magnetic sub-system to temperature $T_{0} . T_{0}$ is the only parameter to control the total energy on the lattice. A second configuration is chosen in the close neighborhood of the first one (Initial distance $D(0)$ is small). Distance $D$ between two configurations is defined as the sum of absolute value of local difference in magnetic energy.

We numerically integrate the equations of motion for both initial configurations and measure the time behavior of their distance $D(t)$. The distance diverges exponentially for the two initially close confiigurations. In Fig.1, the evolution of $\ln (D(t))$ is shown. We see after several initial oscillations, $D(t)$ increases exponentially with $t$ and then saturates at large $t$ due to compact nature of $\mathrm{SU}(3)$ group. We notice in the exponential increasing region the fluctuation is small for large $T_{0}$. This suggests the divergent property is very similar in the entire phase space except for some regions of small measure. We identify the slope of $\ln (D(t))$ in the linear region as the largest Lyapunov exponent $h$.

We shall note here that the divergent behavior is an intrinsic property and it shall not depend on a particular definition of distance we choose for it. This is verified when we choose a slightly different definition and observe the same behavior.

We have also tried to study the wave-length dependence of the trajectory divergence. In this respect the lattice seems to be too small for a conclusive analysis.

After several runs with different initial $T_{0}$ 's, we find a linear relation between $h$ and $E$ shown in fig.2. Here $E$ is the averaged energy on each plaquette. Using the scaling property of the Hamiltonian we get $h a=\frac{1}{10} g^{2} E a$. We see the points fit nicely on the straight line which goes through the origin. In the classical limit, $h$ is independent on $a$ because $g$ does not run with $a$ in this limit. The fluctuations are small because of the large number of degrees of freedom involved. To a good approximation, $E$ is related to the temperature as $E=\frac{16}{3} T$. So finaly we get $h=0.54 g^{2} T$.

We know that the sum of all positive Lyapunov exponents describes the entropy growth rate, which is roughly the inverse of the time scale that the system approaches thermal equilibrium. So the largest Lyapunov exponent leads to an estimate of the thermalization time $\tau_{s}=h^{-1}$. As an example to show the time scale, at $T=400 \mathrm{MeV}, \tau_{s}$ is roughly 0.24 $\mathrm{fm} / \mathrm{c}$. So here we really see that the gluon system thermalizes very fast.

We observe that the numerical value of $h$ and the damping rate nof a gluon at rest is astonishingly close. In the next section we will give some reason for this coinsidence. Though we have not fully understood it, we have proved the necessary base of their relation, i.e. the later is essentially a classical quantity.

To conclude, we have shown numerically that the classical $\mathrm{SU}(3)$ gauge fields are chaotic. This provides an explanation for why the gluon system thermalizes rapidly.

[*] C. Gong, Phys. Lett. B298, 257 (1993).

[1] B. Müller and A. Trayanov, Phys. Rev. Lett. 68, 3387 (1992).

[2] J. Kogut and L. Susskind, Phys. Rev. D11,395 (1975).

[3] E. Pietarinen, Nucl. Phys. B190, 349 (1981). 


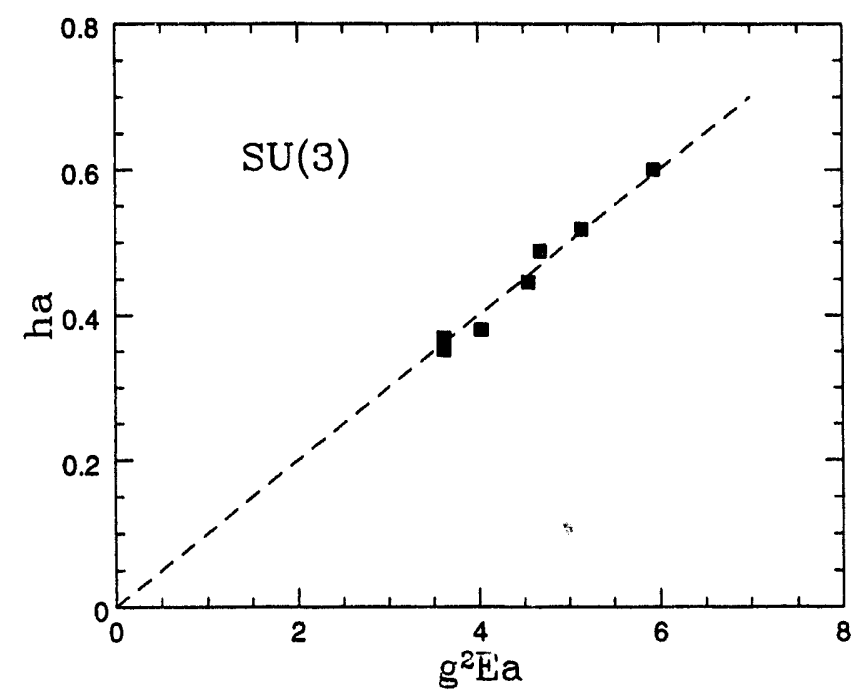

Fig. 2: Maximal Lyapunov exponent in $\mathrm{SU}(3)$ as a function of energy.

\subsection{Classical Nature of Gluon Damping Rate \\ (C. Gong)}

We found in both $\mathrm{SU}(2)$ and $\mathrm{SU}(3)$ the seemingly numerical accident that the value of the maximal Lyapunov exponent $h$ is remarkably similar to twice the damping rate of a rest gluon [1], in a thermal gluon system. This is a quite surprising result because these two quantities Appear in totally different contexts and are calculated with different methods. In the one hand, the damping rate is the imaginary part of the self energy of a quasiparticle in a thermal gluon system and is calculated by a sophisticated effective quantum field theory. While the Lyapunov exponent here is a classical dynamical quantity describing the divergent property of two classical trajectories. But though we can not establish a direct relation between these two quantities in the moment, we think this similarity does not arise without any reason. First these two quantities, though very different from their contexts, both describe how fast a non-equilibrated gluon system approaches thermal equilibrium. The relevance of $h$ is clear from the classical dynamical point of view, where $h$ is directly related to the entropy growth rate. The connection of $\gamma(\omega)$ appears clearly [2]

$$
f(\omega, t)=\frac{1}{e^{\omega / T}-1}+c(\omega) e^{-2 \gamma(\omega) t},
$$

where $f$ is time dependent distribution function, the first term on the l.h.s is the equilibrated distribution. We see $\gamma$ appears in the decaying term. Second, we have proved that the gluon damping rate is basically a quantity of semi-classical origin. Since $\gamma(\omega)$ is a smooth function, it is sufficient to prove the statement for a gluon at rest for which case explicit quantum field calculation is given in Ref. [1]. So in the following, we will concentrate only on the rest gluon. 
The proof basically goes as follows. The damping rate can be obtained as the difference between decaying rate and the production rate, each of which can be calculated by tree level Feyman diagrams suppose we use the effective propagators and vertices $[1,2]$. Furthermore, the later quatities are calculated by tree diagrams using bare propagators and vertices [2]. These diagrams are different from that in the vacuum only by the distribution function factors assigned for each external line. We know a tree level diagram in a massless theory is of classical origin [3]. This leads to the conclusion that the damping rate is semi-classical quantities and the only quantum correction is from the thermal distribution function. If we want to use the classical phase space distribution $n=\frac{T}{\omega}$ in the calculation, we must use a cutoff (lattice size $a$ ) in order to avoid divergence. Then all the effective quatities may depend on $a$. Now to see how $a$ comes into a quantity, we shall express this quantity in terms of classical variables: $g_{c}^{2}=4 \pi \alpha_{s} / \hbar, T$ and $a$. Here $g_{c}$ is so defined that $\hbar$ does not appear explicitly in classical Yang-Mills equations. For example, the plasma frequency will be $\omega_{p}=g_{c} \sqrt{T / a}$, which diverges when a goes to zero. Though $\omega_{p}$ can be calculated semi-classically, it is not well defined at strict classical limit. On the other hand $\gamma(0) \sim g_{c}^{2} T$ is independent on this cutoff $a$ in the leading order. This proves that to the leading order $\gamma(0)$ is a purely classical quantity.

We conclude that the damping rate of a long wave-length mode in the gauge system is purely classical.

[1] E. Braaten and R. D. Pisarski, Phys. Rev. D42, 2156 (1990).

[2] H. A. Weldon, Phys. Rev. D28, 2007 (1982).

[3] C. Itzykson and J. Zuber, Quantum Field Theory (McGraw-Hill, New-York, 1.980).

\subsection{Lyapunov Spectra in Gauge Theory by Rescaling Method (C. Gong and B. Müller)}

Though the previous method is easy to use to obtain the largest Laypunov exponent of a given theory, there are two drawbacks. First the value thus obtained has a large error bar because the exponential divergence of trajectories has fluctuations, which results in an uncertainty in the determination of the exponential rate. The second drawback of the method is that only the largest Lyapunov exponent can be obtained, but not the whole Lyapunov spectra. Here we introduce a new method which can determine Lyapunov exponents more precisely and whcih in principle can be used to obtain the whole Lyapunov spectra.

This method is widely used in stuuying chaotic dynamical systems of few dimensions [1], which we call rescaling method here. Suppose we want to calculate the largest two Lyapunov exponents of the system. We can randomly choose three initial points, to which we refer as $z_{0}(0), z_{1}(0), z_{2}(0)$ for convienince, in the phase space, with the condition that they are close to each other according to some appropriate distance measure. If the system is chaotic, or if the initial points are chosen inside the chaotic part of the phase space, the distances between the three trajectories $z_{i}(t)$ evolved from the three initial points will diverge exponentially. Let us denote the distance between trjectory $z_{i}(t), i=1,2$ and $z_{0}(t)$ as $d_{i}$ and the distance between $z_{1}$ and $z_{2}$ as $d_{3}$. Since the available phase space volume 
is limited by energy, $d_{i}$ will saturate after relatively short period. To avoid saturation, the following rescaling method is used. A fixed reference distance $d_{0}$ is given. The whole procedure consists two steps. In the first step, trajectories $z_{i}(t)$ evolve according to the equations of motion for a period of time $t_{0}$. Then in the second step we rescale the distances $d_{1}$ and $d_{2}$ as follows. We have point $z_{0}(t)$ fixed, while distance $d_{1}$ is scaled back to $d_{0}$ by bringing $z_{1}$ to $z_{0}(t)$ along the direction $d_{1}(t)$. The scaled factor is denoted $s_{1}^{k}$, where $k$ refers to the $k$ th rescaling. For $z_{2}(t)$, we first orthogonalize $d_{2}$ against $d_{1}$ then scale the orthogonalized $d_{2}^{\prime}$ to the refernce value $d_{0}$. The scale factor is denoted $s_{2}^{k}$. This procedure is to be iterated $N$ times until the Lyapunov exponent,

$$
\lambda_{i}=\lim _{N \rightarrow \infty} \sum_{k=1}^{N} \frac{\ln s_{i}^{k}}{t_{0}},
$$

converges. Here $\lambda_{1}$ is the largest Lyapunov expoenent and $\lambda_{2}$ is the second largest one. In principle this method can be used to obtain the whole Lyapunov spectra accurately. The time needed to obtain the larest Lyapunov exponent depends on how fast the exponents converge with increasing $N$.

In the first glance, the applicability of this method to lattice gauge system is doultful. The reason is that while the meaning of rescaling and orthogonalization is quite obvious in a Euclidean phase space, it is not less clear in the case of a lattice gauge system, where the phase space is curved as well as constrainted by Gauss' Law. The problem of a curved space is relatively simple. One possible way is to transform link variable $U_{l}$ back to vactor potential $A$, so we can work in the phase space formed by electric fields and vector potentials, for which the geometry is simply Euclidean. In the case of SU(2), we have yet another simpler method. Each SU(2) group element is represented by a normalized quaternion. When performing orthogonaling and rescaling, We can simply treat all the 4 components of the quaternion as independent cartesian coordinates, because locally the metric of the curved space is Euclidean. Now we come to Gauss' Law. Actually it does not pose strong constraint on our method. The reason is as follows. When we rescale the variables, we indeed violate the Gauss' law. But if the distance $d$ is small, then the violation of Gauss' law is of the second order of $d$. So if we limit ourselves to a small $d$, then the violation of Gauss's Law in each step is negeligible. Also we observe that the evolution of the system respect Gauss' Law, so the violation does not increase in time. The next rescaling decreases this violation by a scale factor, so the violation does not accumulate.

We have used this method to study $\mathrm{SU}(2)$ system and measured the largest two Lyapunov exponents. We indeed see that the Gauss' Law violation is only of the order of $10^{-6}$. The result for Lyapunov exponent of a typical run is shown in Fig.1. The corresponding energy is $g^{2} E a=4.06$. The solid line corresponds to the largest exponent and the dotted is the second largest one. They converge at $t \approx 100$. Note the time scale of saturation in the case without rescaing is about 30 at the same energy. $\lambda_{0}=0.667$ is very close to our previous result $4.06 / 6=0.677$, where we do not use the rescaling. We also observe $\lambda_{1}$ is almost identical to $\lambda_{0}$. 


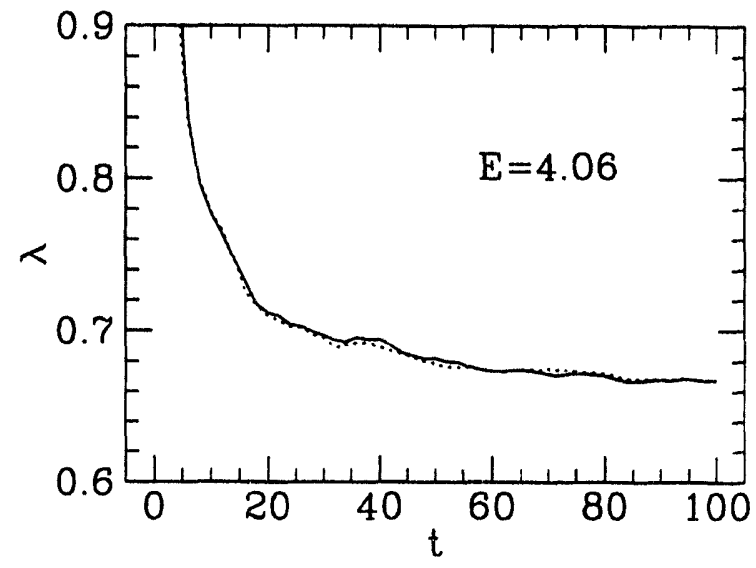

Fig. 1: The first two Lyapunov exponents of a SU(2) lattice gauge fields. The solid line is for the larest Lyapunov exponent and the dotted line is for the second largest one.

The future work is to extend the calculation to cover all the positive Lyapunov exponents in order to fully understand the dynamics of the system.

[1] see for example, G. Benettin, C. Froeschle, and J. P. Scheidecker, Phys. Rev. A19, 2454 (1979).

\subsection{Chaoticity in Massless Higgs Fields and Massive Gauge Fields (C. Gong, S. G. Matinyan and A. Trayanov)}

We have applied the above method to other two systems, i.e., the massless Higgs fields and the massive gauge fields, to study some related problem.

One question concerning the chaoticity of non-abelian gauge fields is that whether the chaoticity is just a consequence of non-linearity, or it is related to the particular type of non-abelian gauge interaction. In this respect, we want to study the classical $\phi^{4}$ theory on the lattice. The lattice formulism of a interacting Higgs and gauge system is seen in Ref.1. We here study a spe sial case, i.e. the massless limit of a doublet Higgs field, which has 4 real field components. In the massless limit, just like in the case of a gauge theory, the corresponding classical lattice theory is scale invariant, in the sense that the coupling $g^{2}$ (usually it is written as $\lambda$ ) and lattice spacing $a$ can be scaled out entirly and the system does not have any parameter except the total energy. Using the same method as for the gauge system, we measured Lyapunov exponents of the massless Higgs fields on the lattice. In Fig.1 we show the $\lambda a / g^{2} E a \sim g^{2} E a$ relation. First we observe that the Layapunov exponents in Higgs fields is much smaller to that in gauge fields with the same energy. Second, we find at small $g^{2} E a$, the ratio $\lambda a / g^{2} E a$ tends to zero, which suggests a vanishing Lyapunov exponent in the continuum limit when $a \rightarrow 0$. This result is consistent to our understanding of the relation between the maximal Layapunov exponent and the damping rate of the long wave length modes. The thermal perturbative calculation shows, unlike in the case of non-abelian gauge fields, the dainping rate of the long wavelength mode 
in the Higgs field vanishes at the order of $g^{2}$. This study shows that not all non-linearity leads to chaos.

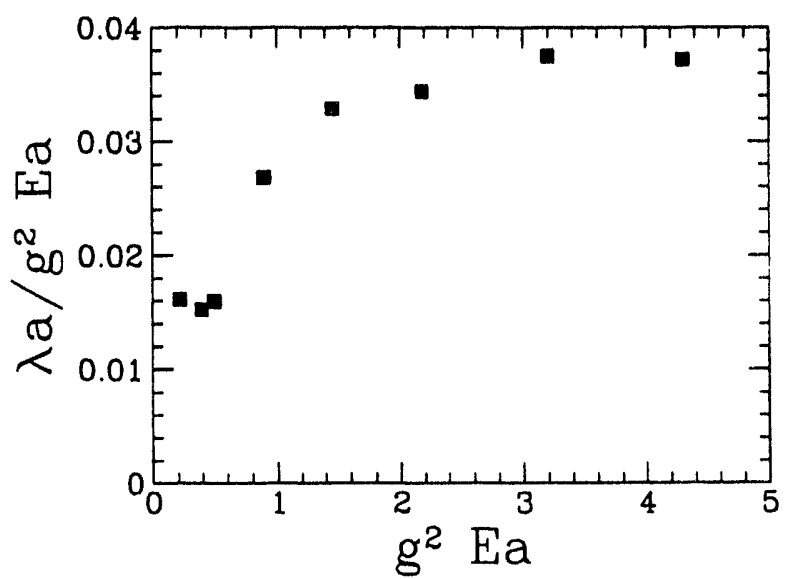

Fig. 1: Lyapunov exponents of a massless Higgs theory.

We have also studied the massive SU(2) gauge fields. This is the first step toward the understanding of chaoticity in electro-weak interaction, where the gauge symmetry is spontaneously broken by a Higgs field. We want to study how a mass term affects the chaoticity, which will also give some hint on how the interaction between Higgs field and the gluon field affects the chaoticity of the later. In Fig.2, we show the largest Lyapunov

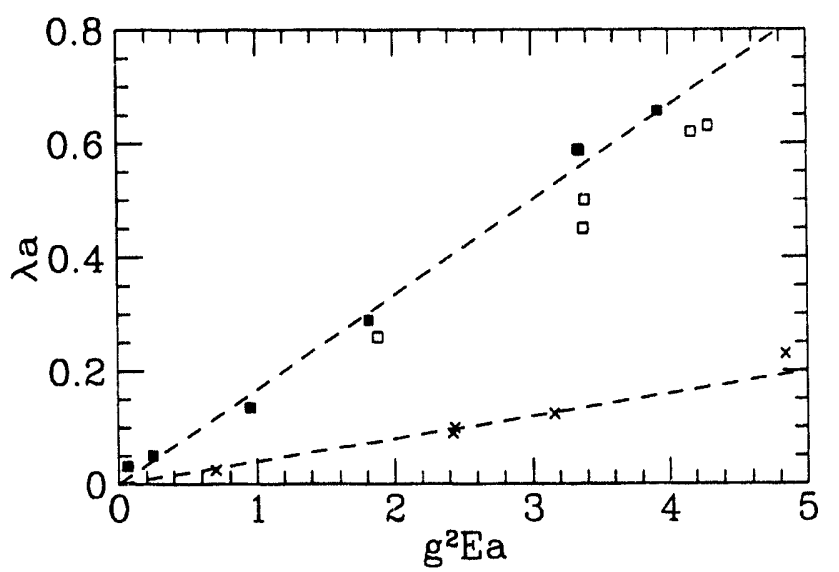

Fig. 2: Lyapunov exponents of massive gauge theories. The solid are for $m=0$, the hollow are for $m=0.2$ and the cross are for $m=4$.

exponents at different energies for theories with different mass. The same scaled variables are used, but here the mass parameter $m$ cannot be scaled out. $m$ is related to the mass $M$ of the gauge fields in the continuum theory as $M=\frac{\hbar}{2 a} m$. For comparison, the data for the massless theory are shown as solid sqaures, which are fit by a straight line with a coefficient of $1 / 6$. The hollow ones are for $m=0.2$ and the cross ones are for $m=4$. They more or less lie on a straight line. We observe the effect of a mass term is to reduce the chaoticity of the system, 
or in another words, the mass has a stabilizing effect. This effect is consistent to our intuition from studies on the coupled harminic oscillator, where the strength of chaoticity depends on the relative strength of the non-linearity and the mass [1].

[1] S. G. Matinyan, G. K. Savvidy and N. G. Ter-Arutyunyan-Savvidy, Sov. Phys. JETP 53, 421 (1981); JETP Lett. 34, 590 (1981).

\subsection{Gaussian Wavepacket and Quantum Corrections in SU(2) (C. Gong, B. Müller, T. Biró)}

After studying the classical limit of the gauge system, it is natural to ask how relevant this kind of investigation is. Or in another word, we want to have some understanding on the quantum corrections to our classical results. As our first attack on this problem, we apply a variational method to Gaussian wave packets. This method is widely used in investigating both static and initial value problems in various fields, where the ordinary perturbative method is not applicable $[1,2,3]$. Here we are interested in solving initial value problems. The basic idea is to parametrize a wave packet, usually a Gaussian one, by a small number of parameters. Then the time dependent variational principle is used to derive a set of evolution equations for these parameters, which have a symplectic structure like Hamiltonian equations. If the parameters are chosen in such a way that some of them resemble the variables in the corresponding classical system, then the coupling between the semi-classical time evolution of these parameters and that of all others can be interpreted as quantum corrections to the classical evolution.

We have chosen the following Gaussian ansatz,

$$
\Phi\left[U_{l}\right]=\prod_{l} \phi_{l}\left(U_{l}\right)=\prod_{l} \frac{1}{\sqrt{N_{l}}} \exp \left(\frac{b_{l}}{2} \operatorname{tr}\left(U_{l} U_{l 0}^{-1}\right)-\frac{1}{\hbar} \operatorname{tr}\left(E_{l 0} U_{l} U_{l 0}^{-1}\right)\right)
$$

The $U_{l 0}$ and $E_{l 0}$ are parameters of the wavefunction, which correspond to classical link variables and left electric fields, respectively. The $U_{l}$ 's and $U_{l 0}$ 's are group elements of $\mathrm{SU}(2)$, where as the $E_{l 0}$ are elements of the $\mathrm{su}(2)$ algebra. The complex parameter $b=v+i w$ controls the width, or more explicitely, $\sqrt{v}$ is the inverse of the width. $N_{l}$ is a normalization constant. Wavefunctions with different parameters are not orthogonal to each other. They form a set of overcomplete basis states of the Hilbert space for each link.

The wavefunction we chose is separable in link variables. In the strong coupling and zero temperature limit, the ground state wave function is an expansion of plaquette variables $\operatorname{tr} U_{p}[4]$. It is separable for different plaquettes, but this choices is not very practical in our context. First we want the trial function to have the feature that the averages can be performed in closed analytical expressions. A plaquette separable wavefunction is more difficult in this aspect than 
a link separable one. The second reason is that we are mainly interested in the high temperature limit where the system can be treated classically. In this limit the former is not a priori better justified than the latter. Moreover, since in the classical limit we deal with link variables rather than plaquette variables, a link separable wavefunction allows to make a more direct connection to classical dynamics.

The quantum variations of electric fields and vector potentials are obtained. ¿From the requirement that the quantum variations shall be smaller than the classical distances for a classical discription to make sense, we obtain the conditions: (1) $\frac{\hbar}{T a} \ll 1$, and $(2) \hbar \ll 1$.

The effective Hamiltonian is obtained as the expectation value of the Hamiltonian operator under the Gaussian ansatz,

$$
\begin{aligned}
H_{s c} & =H_{e}+H_{0}+H_{m} \equiv\langle H\rangle \\
& =\sum_{l}\left[\frac{1}{2}\left(1-\frac{f_{l}}{2 v_{l}}\right) E_{l 0}^{2}\right]+\sum_{l}\left[\hbar^{2} \frac{3 f_{l}}{16 v_{l}}\left(v_{l}^{2}+w_{l}^{2}\right)\right]+4 \sum_{p}\left(1-\frac{1}{2} f_{p} \operatorname{tr} U_{p 0}\right),
\end{aligned}
$$

where $f_{l}$ is a function of $v_{l}$ which cotains corrections due to finite width and goes to 1 in the limit of vanishing width. This semi-classical Hamiltonian consists of three parts. First we have the modified electric energy $\left(H_{e}\right)$ and magnetic energy $\left(H_{m}\right)$, which result in the coupling between the $U_{l}, E_{l}$ and the width parameters $v_{l}$ and $w_{l}$, and thus modify the classical evolution of $U_{l}$ and $E_{l}$. These modifications contain both genuine quantum effects and lattice artifacts, which are difficult to separate. Besides the modified classical electric and magnetic energy there is a zero point energy $\left(H_{0}\right)$ which is a consequence of the uncertainty relation. It increases linearly with $v$

The semi-classical equations of motion are obtained from the following variational method,

$$
\delta \int_{t 1}^{t 2}\left\langle\Phi\left|\left(i \hbar \partial_{t}-H\right)\right| \Phi\right\rangle=0 .
$$

In the limit of small $\hbar$ these semi-classical equations assume the correct classical form. The differences will result in quantum corrections. We note here the Gauss's Law is conserved averagely by evolution in this formalism.

With the above semi-classical equations of motion we can follow a trajectory in the space of gauge fields for every given initial condition. To make the results comparable to those of our calculations for the classical gauge theory, we specify the initial gauge configuration in the same way as in ref.[1]. We initially choose all electric field variables to be zero to satisfy Gauss's law. The magnetic sector is initialized by chosing the link variables $U_{l}=\cos (\rho / 2)-i \mathbf{n} \cdot \tau \sin (\rho / 2)$ limits the range of the parameter $\rho$ to $(0,2 \pi \delta)$. In addtion here we specify $\hbar$ and the width parameter. Initially we choose identically on each link $v=20$ and $w=0$. The results are given in Table 1.

To present the result we need to specify our controllable parameters. One of these is $\hbar=4 \pi \alpha_{s}$, which is a manifestation of quantum effects. Another 
should be chosen to give a measure of the excitation energy of the system. A direct comparison between classical and quantal systems with the same energy is unphysical because of the appearance of a zero-point energy in the latter. A convenient comparable parameter is the physical into a phase space prefactor and an exponential $\exp (-E / T)$. Dividing out the phase space factor we can simply identify the temperature as the rate of exponential slope of the energy distribution. But here this method cannot be used directly because the lattice gauge system is a constrained and strongly coupled system. This problem will be discussed in detail elsewhere. Here we make the observation that the relative difference between the electric part of the semi-classical Hamiltonian and that of the classical one is $1 /(2 v)$ which is very small in the limit of large $v$. As a consequence, the value of $\left\langle\left\langle H_{e}\right\rangle\right\rangle$, the average electric energy per link, at a certain temperature $T$ of the classical system and that of the semi-classical system in the limit of large $v$ will be similar. Neglecting the small difference, we can use it as a measure of the excitation level of the system, and define parameter $T_{e} \equiv\left\langle\left\langle H_{e}\right\rangle\right\rangle$ as the "electric temperature" of the gauge field.

First the behavior of average $v$ and $w$ with tim is studied. Both of them reach stable values after some osscilation. The stable value of average $w$ is zero. The average $v$ has a simple form $7 / \hbar$. We have also studied the chaoticity using these equations and compared to that in the classical limit. In Fig.1, we show the divergence of trajectories with time. The solid line is with quantum correction and the dotted one corresponds to the classical limit with same temperature. We see the distance in the quantum case rises more rapidly. This is observed for all initial conditions we have studied.

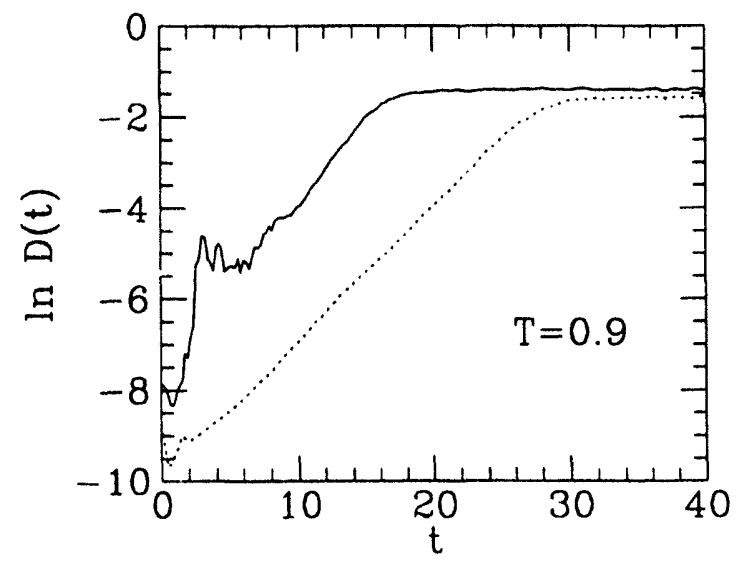

Fig. 1: The solid line shows the divergence of the centroids for the two neighbouring Gaussian field configurations for $T_{e}=0.9$ and $\hbar=0.3$. The corresponding divergence for the two classical field configurations with the same $T_{e}$ is shown by the dotted line.

We conclude that the quantum corrections enhance the chaoticity in the non-abelian gauge system. 
Table 1

\begin{tabular}{cllllr}
$\hbar$ & $T$ & \multicolumn{1}{c}{$\lambda$} & $\delta \lambda$ & $\langle\langle v\rangle\rangle$ & $E$ \\
0.1 & 0.76 & 0.255 & 0.01 & 70 & 2.13 \\
0.3 & 0.64 & 0.24 & 0.12 & 23 & 2.48 \\
0.3 & 0.73 & 0.3 & 0.22 & 22 & 2.92 \\
0.3 & 0.85 & 0.34 & 0.2 & 21 & 3.31 \\
0.3 & 0.85 & 0.36 & 0.27 & 21 & 3.25 \\
0.3 & 0.85 & 0.34 & 0.2 & 22 & 3.25 \\
0.3 & 0.9 & 0.41 & 0.37 & 20 & 3.74 \\
0.7 & 0.5 & 0.19 & 0.16 & 9 & 3.24 \\
0.7 & 0.8 & 0.41 & 0.54 & 7.4 & 4.40
\end{tabular}

[1] M. Saraceno, P.Kramer, and F. Fernandez, Nucl. Phys. A405 88 (1983).

[2] H. Feldmeier, Nucl. Phys. A515, 147 (1990).

[3] C. Corianó, R. Parwani and H. Yamagishi, Nucl. Phys. A522 (1991) 591.

[4] S. A. Chin, O. S. Van Roosmalen, E. A. Umland and S. E. Koonin, Phys. Rev. D31, 3201 (1985).

\subsection{Thermalization in $\mathrm{SU}(2)$ Gauge Theory}

\section{(A. Trayanov)}

Studies of the thermalization properties of quark gluon plasma add an important contribution to the global understanding of the processes that occur in heavy ion collision experiments. Recently it has been demonstrated that the nonabelian gauge fields exhibit semi-classical chaotic dynamics. The chaoticity of the gauge fields means that the classical Yang-Mills system is ergodic. The time evolution of such system creates a microcanonical ensemble of a given energy. The gauge fields thermalize themselves due to the non-linear self-interaction which is a result of the non-abelian nature of the gauge group. The universal exponential divergence of neighboring gauge field implies that the entropy of an ensemble of gauge fields grows linearly with time until the available microcanonical phase space is filled and the system is equilibrated. To estimate the time needed for thermalization we substitute an approximate relation between the average energy $E$ per plaquette and temperature in the expression for the rate of entropy growth as function of the total enery

$$
E \approx 2 T[S U(2)], \quad E \approx \frac{16}{3} T[S U(3)] .
$$

Then the characteristic entropy growth rate is given by

$$
\Gamma \equiv h \approx\left\{\begin{array}{lll}
0.34 g^{2} T & {[\mathrm{SU}(2)]} \\
0.54 g^{2} T & {[\mathrm{SU}(3)]}
\end{array}\right.
$$


Apart from a factor two, these values are remarkably close to those obtained for the thermal gluon damping rate by Braaten and Pisarski.

The thermalization of a real physical system occurs via energy exchange by absorption and emission of field quanta. To simulate this process on a computer, one needs to couple the lattice gauge system to an external thermal bath which will allow for energy exchange. Several schemes are possible and the basic idea is to use a Monte Carlo technique, which will accept or reject a set of new generated link variables and their time derivatives according to a thermal distribution. For an $\mathrm{SU}(2)$ gauge system the most efficient way is the so-called thermal bath algorithm developed by Creutz, which significantly reduces the rejection rate in the Monte Carlo process. However, a straightforward application of this method has the disadvantage that it violates Gauss' law. Generating either a new link variable or its time derivative changes the electrical field associated with that particular link. In order to satisfy the Gauss law one has to adjust, the U's or $\dot{U}$ 's on all the other links. This would require solving of $9 L^{3}$ coupled equations for $\mathrm{SU}(2)$, which, of course, is not practical. The only case when the thermal bath method would have a practical significance is the case when there is no electric field.

Fortunately we can make use of the ergodicity of our system. Therefore we can locally thermalize, let us say, the magnetic field, and employ the time evolution to distribute the excess or deficiency of energy between all degrees of freedom. After relatively short period of time the initially given energy is distributed between the electric and magnetic energies.

Several questions need to be answered: how do we know in practice that the system has reached a thermal equilibrium; and if it has, what is the actual temperature and how to measure it. To answer those questions we studied the details of the thermalization process i pure gauge system by both Monte Carlo heat bath method and time-evolution induced thermalization.

First we start with a system with no electric field. Since the Gauss' law is always satisfied for such a system the original Creutz's heat bath method can be used. After performing several thousand Monte Carlo heat bath thermalization steps per plaquette we plot the distrubution of the magnetic energy for different bath termperatures (Figure 1). In the case of $\mathrm{SU}(2)$ gauge group the group measure can be accounted for analytically and the plot can be linearized in semilogarithmic coordinates. As the figure shows the distribution is linear for all temperatures. The plot deviates from linearity only at high energies due to small number and poor statistics of these rarely populated configurations. The straight line is clear indication that all degrees of freedom are equally populated, i.e., the gauge system is in thermal equilibrium. However, each of the straight lines has a slope which differs from the value $1 / T$ which is expected from the Boltzmann distribution. The reason for such a behavior is that the number of effective degrees of freedom changes with the system temperature. 


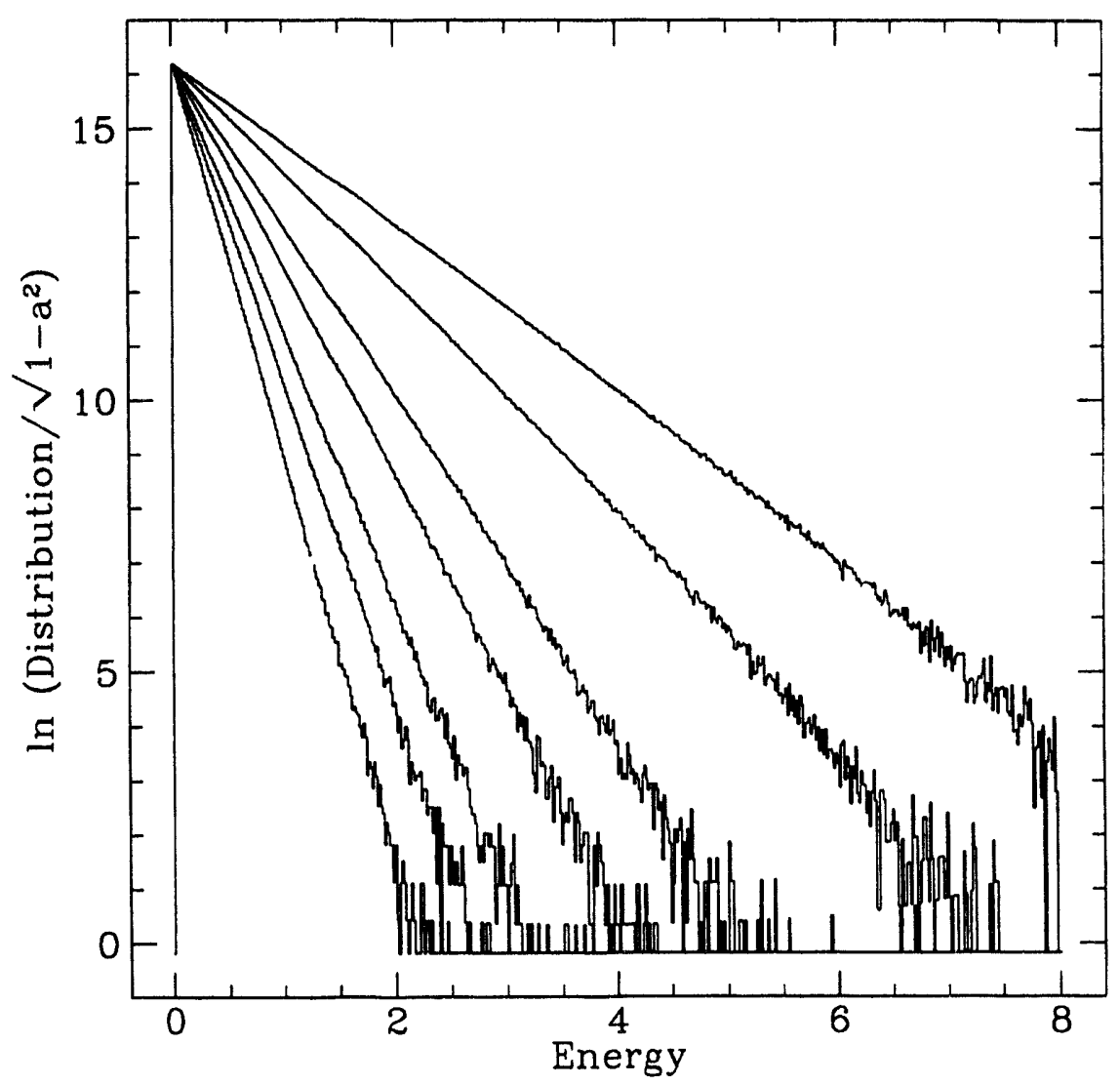

Fig. 1: Distribution of the magnetic energy

On Figure 2 we plot the slopes of the distributions from Figure 1 as a function of the bath temperature. We can use the data from the plot as a "thermometer", i.e. if we want to measure the temperature of the gauge fields, we need to plot the distribution of the magnetic gauge energy, and the from its slope, using Figure 2 find the corresponding temperature. At high temperatures $(T>>2)$ the temperature from the Boltzmann distribution coincides with the bath temperature while at low temperatures some degrees of freedom are "frozen" and their number tends to $1 / 3$ at very low temperatures.

Finally we apply the outlined method to verify that the dynamical evolution leads to thermal equlibrium and find the the temperature in the system. In the classical description of the gauge system the electric field is equvallent to momentum in a mechanical system and therefore a simple degrees-of-freedom counting argument would predict that the average electric energy would be equal to $T$ (for SU(2)). As expected, a short time evolution $(t \sim 1)$ was enough to redistribute the total energy among all degrees of freedom. Our findings also confirmed the expected values for the electric energy.

[1] A. Trayanov and B. Müller, in Computational Quantum Physics, edited by A. S. Umar et al. (AIP, New York, 1992), p.552 
[2] M. Creutz, Quarks, gluons and lattices, Chap. 13 (Cambridge University Press, Cambridge 1983).

[3] B. Müller and A. Trayanov, Phys. Rev. Lett. 68, 3387, (1992)

[4] E. Braaten and R. D. Pisarski, Phys. Rev. D42, 2156 (1990).

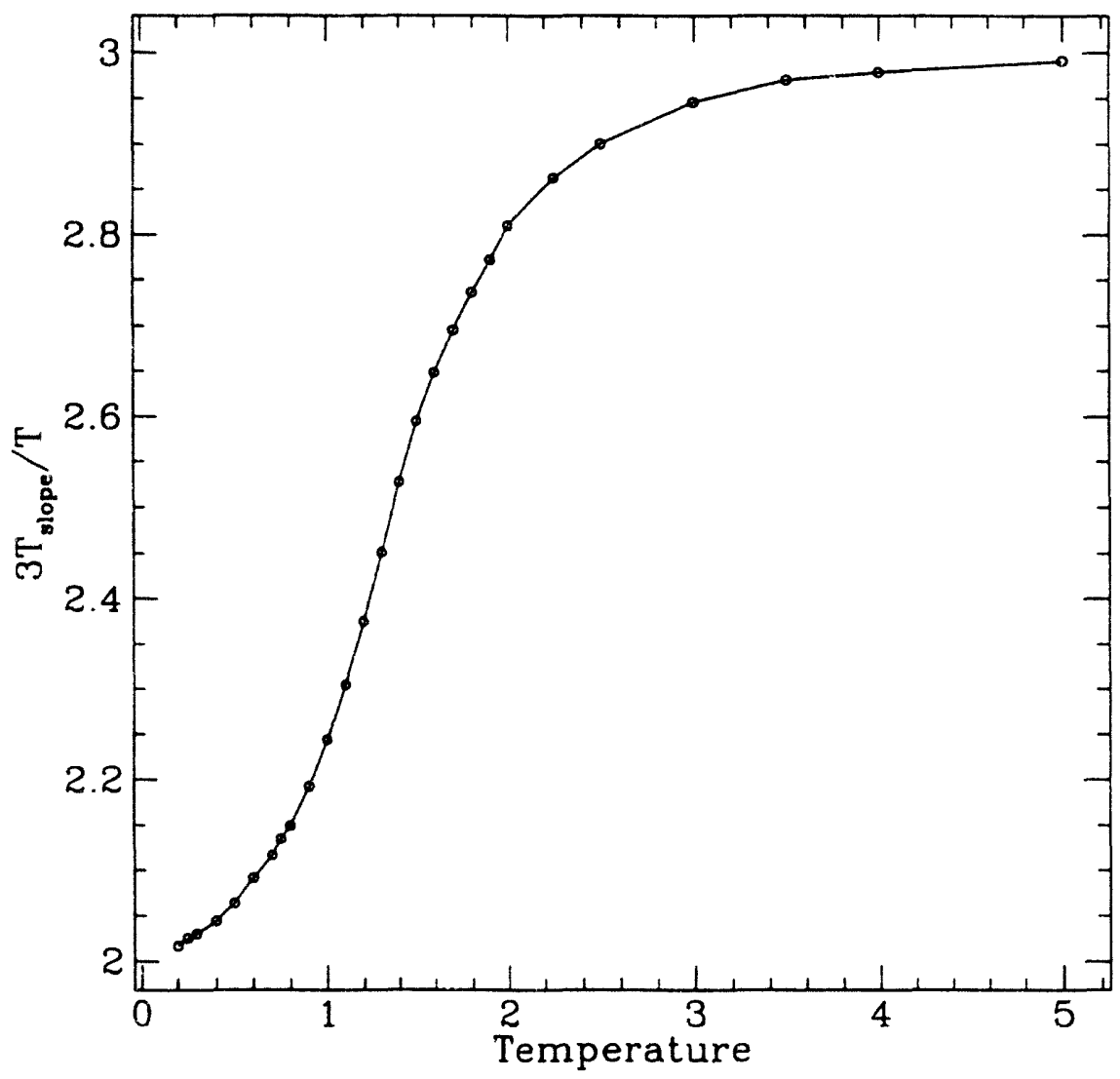

Fig. 2: Slopes of the distributions from Figure 1 as function of temperature

\subsection{Real-Time Evolution in the Non-Linear Sigma Model}

(A. Trayanov)

The properties of several models from the statistical physics have been debated for a long time in the literature, especially some of the aspects relevant to the theory of phase transitions. Recently there has been an renewed interest in the so-called non-linear sigma model with emphasis of its applications to pion physics and the physics of heavy ion collisions. In this model the pion field is represented by a four component vector field, $U \equiv(\sigma, \vec{\pi})$, which, in analogy with physics of the ferromagnets, can be called a generalized 4-dimensional "spin". In the lattice formulation the fields are defined at each lattice point and the Hamiltonian has the following form

$$
H=\frac{1}{2} \sum_{\ell} \dot{U}_{\ell}^{a} \dot{U}_{\ell}^{a}+V(U)+V_{e x t}
$$


Here $V(U)$ is the potential of pion interaction. Our main interest will focus on the case of 4-dimensional "spins" on a 3-dimensional lattice, where the spin variables represent the Higgs field. In the continuum limit the low energy interactions of the pions are expressed as

$$
V(U)=M(\nabla U)^{2}+\lambda U^{4}+\text { high order terms }
$$

The external field $V_{\text {ext }}$ represents the pion mass term, and it has the form

$$
V_{e x t}=\frac{1}{2} \operatorname{trh} \cdot U^{+}
$$

We will limit the discussion to the "linear sigma model", i.e., neglecting all the terms but the first one in the above equation. In this case the $U$-variables have unit length, i.e. $\left(U_{\ell}^{a}\right)^{2}=1$. The lattice Hamiltonian becomes

$$
H=\frac{1}{2} \sum_{\ell} \dot{U}_{\ell}^{a} \dot{U}_{\ell}^{a}+M \sum_{\ell, j} \operatorname{tr} U_{\ell}^{a} U_{\ell+j}^{a}+\frac{1}{2} \sum_{\ell} \operatorname{tr} h \cdot U_{\ell}^{+}
$$

where summation over $j$ spans the nearest neighbors of the site $\ell$. The static version of this model $\left(\dot{U}_{\ell}^{a} \equiv 0\right)$ is known in the statistical and condensed matter physics as Heisenberg model. The 4 dimensional spins on a 3 dimensional space lattice have a first order phase transition transition at temperature $T_{c} \approx 1.43 \mathrm{M}$.

Our main interest in this work is the dynamics of the pion field. To study the problem we first have derived the equations of motion from the Hamiltonion (eq.3) in the semi-classical approximation.

$$
\ddot{U}_{\ell}^{a}=\sum_{j}^{n g b} U_{\ell+j}^{a}-\left\{\frac{1}{2} \operatorname{tr}\left[\left(\sum_{j}^{n g b} U_{\ell+j}\right) U_{\ell}^{+}\right]+\frac{1}{2} \operatorname{tr} \dot{U}_{\ell} \dot{U}_{\ell}^{+}\right\} U_{\ell}^{a}-h\left(U_{\ell}^{+}-\frac{1}{2} \operatorname{tr} U_{\ell}^{+}\right) U_{\ell}^{a}
$$

The next step is to numerically integrate the equations of motion. We have developed a computer code to perform the Hamiltonian evolution. The numerical integration is based on the fourth-order-accurate Runge-Kutta method. For purposes of more efficient use of the memory and the CPU, the pion fields are represented as quaternions.

Figure 1 shows the dynamics of the $\sigma$-components of the pion field. The field oscillates with a specific frequency and tends to orient in such a way that $t$ would minimize the contribution of the mass term. 


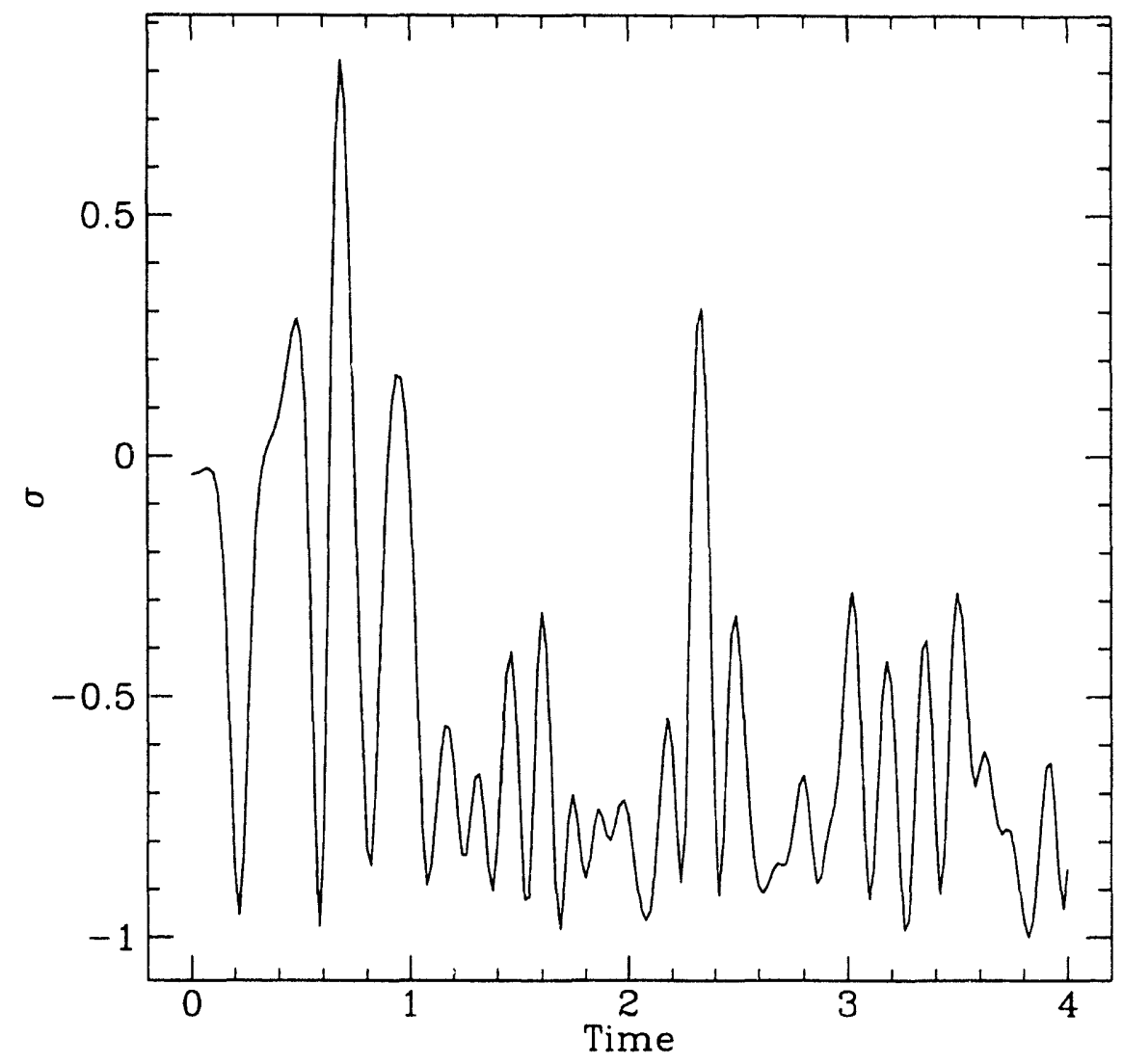

Fig. 1: Dynamics of the $\sigma$-component of the pion field

[1] J. Ambjorn, T. Askgaard, H. Porter and M. E. Shapoc'innikov, Nucl.Phy. B353, 346, (1992)

[2] K. Rajagopal and F. Wilczek, preprint PUPT 1389 (1993) 


\section{Studies In QuANTUM Field Theory}

\subsection{Irreversibility in Quantum Theory}

\section{(J. Rau)}

Experiments in high energy physics, such as collisions of elementary particles or heavy ions, confront us with a startling paradox: While the initial state of the system (before the collision) is almost pure, particles emerging from the reaction can very well be described by a thermal distribution. This thermalization is accompanied by a large apparent increase in entropy, indicating that the process must be highly irreversible; yet at the same time the entire reaction is governed by the laws of quantum mechanics which are invariant under time reversal. (Violations of microscopic time reversal symmetry due to weak interaction are not considered here.) This is just a special case of the so-called irreversibility paradox that has plagued physicists since the early days of thermodynamics [1]. Indeed, if the statistical operator evolves according to the Liouville-v. Neumann equation then the v. Neumann entropy of a state $\rho$, defined as

$$
S_{\mathrm{v} . \mathrm{N} .}:=-k \operatorname{tr}(\rho \ln \rho),
$$

remains constant. Clearly, the v. Neumann entropy cannot be the appropriate quantity for the description of irreversibility; it differs from the 'relevant' entropy whose increase one observes. The v. Neumann entropy and the relevant entropy refer to two different levels of description, called microscopic and mesoscopic, respectively. The transition from the microscopic to a mesoscopic level of description amounts to a coarse graining. Inevitably, information about certain degrees of freedom gets thereby lost.

In the mesoscopic description, one retains information only about some selected observables which are deemed relevant. For the set of 'relevant observables' there are, in principle, many possible choices, each choice corresponding to a different level of description and consequently to a different relevant entropy. It would be obvious to consider those observables relevant which are actually being measured in the experiment. More important, however, and independent of the particular experimental set-up, is the so-called 'Markovian' level of description which is comprised of all slowly varying observables. Typically, this Markovian level is an extension of any experimental level, because observables often have to be slow in order to be measurable in practice. The Markovian level is well defined only if the system exhibits a clear separation of time scales.

Each level of description gives rise to a statistical prediction problem: given the present expectation values of the relevant observables, and possibly their past history, can one predict their future values? On the microscopic level, this prediction problem can be solved - at least formally - by unitary evolution. On mesoscopic levels, however, predictions generally depend in a complicated manner on both the present values and the past history of the relevant observables. How far back into the past one has to reach in order to make a prediction is determined by the memory time. Only if this memory time is small compared to the typical time scale on which the relevant observables evolve, can memory effects be neglected and predictions for the relevant observables be based solely on their present values. This approximation is the Markovian limit; which, in general, is justified 
only on the Markovian level of description. Clearly, a prediction cannot possibly contain more information than the data on which it is based. Thus in the Markovian limit, future expectation values of the relevant observables cannot carry more information than their present values (based on which they could be predicted). Applied iteratively, this means that the amount of information carried by the relevant observables can never increase; or, that the corresponding relevant entropy can never decrease-which is the H-theorem. It is important to note that the $\mathrm{H}$-theorem, i.e., the increase of the relevant entropy, is a consequence of the Markovian limit and as such only applies to the Markovian level of description [3].

While the constancy of the v. Neumann entropy shows that the full (microscopic) description keeps its information content, the growth of the relevant (Markovian) entropy indicates that the amount of information carried by the slowly varying observables steadily decreases. In the course of time, information about the system is being transferred from slow to fast degrees of freedom. This flow of information is perceived as irreversible. But the information is not lost. It only becomes inaccessible to a certain level of description.

Understanding the thermalization properties of a physical system is thus entirely a matter of finding the Markovian level of description, and hence of identifying time scales.

Any study of irreversible phenomena must proceed by:

identifying all time scales,

finding the Markovian level of description, and

finding all conservation laws and thus all constants of the motion.

This knowledge can be used to obtain:

the rate of increase of the relevant (Markovian) entropy, and

the equilibration time (provided the thermalization is complete).

The time scales, and hence the thermalization properties, of a system generally depend on:

features of the dynamics (i.e., the form of the interaction Lagrangian),

coupling constants, and

macroscopic parameters (e.g., density, pressure, etc.).

[1] Balian, R., From Microphysics to Macrophysics, Vols. I,II. Berlin, Heidelberg, New York: Springer 1991,1992

[2] Jaynes, E.T., Am. J. Phys. 33, 391 (1965)

[3] Fick, E., Sauermann, G., The Quantum Statistics of Dynamic Processes, Berlin, Heidelberg: Springer 1990

\subsection{Entropy Production in Vacuum Decay}

(J. Rau)

The decay of a single resonance $|i\rangle$ weakly coupled to a continuum of states $\{|\omega\rangle\}$ is a prime example for an irreversible quantum process. Despite its simplicity, it exhibits all the essential features of a non-equilibrium process. The Hamiltonian is of the form

$$
H=H^{(0)}+\alpha V,
$$


the perturbation having non-vanishing matrix elements

$$
V_{i \omega}=\langle i|V| \omega\rangle=\langle\omega|V| i\rangle^{*}
$$

For simplicity, all other matrix elements of $V$ are taken to be zero. One wishes to describe the time evolution of the occupation probability

$$
p(t):=|\langle i \mid \psi(t)\rangle|^{2}
$$

given that the system is initially in the resonance state.

Employing the so-called projection method pioneered by Nakajima, Zwanzig and others $[1,2]$, one obtains an integro-differential equation for the occupation probability:

$$
\dot{p}(t)=-\alpha^{2} \int_{0}^{t-t_{0}} \mathrm{~d} \tau p(t-\tau)\left\langle\left\langle i\left|\mathcal{V} \mathcal{V}^{I}(\tau)\right| i\right\rangle\right\rangle
$$

with

$$
\left\langle\left\langle i\left|\mathcal{V} \mathcal{V}^{I}(\tau)\right| i\right\rangle\right\rangle=\frac{2}{\hbar^{2}} \operatorname{Re} \int \frac{\mathrm{d} \omega}{2 \pi}\left|V_{i \omega}\right|^{2} \exp \left[i\left(\omega-\omega_{i}\right) \tau\right]
$$

In general, the resonance couples to an entire 'band' of continuum states. This band has some width $\Delta$, called the coupling width. For the sake of simplicity, let us parametrize the coupling as a Gaussian distribution

$$
\left|V_{i \omega}\right|^{2}=\frac{v^{2}}{\Delta} \exp \left[-\frac{\left(\omega-\omega_{i}\right)^{2}}{2 \Delta^{2}}\right]
$$

normalized such that $\operatorname{tr}\left(V^{2}\right)=\sqrt{2 / \pi} v^{2}$. Then the frequency integration may be performed, yielding another Gaussian distribution for $\tau$ with width $\Delta^{-1}$ :

$$
\left\langle\left\langle i\left|\mathcal{V} \mathcal{V}^{I}(\tau)\right| i\right\rangle\right\rangle=\frac{v^{2}}{\hbar^{2}} \sqrt{\frac{2}{\pi}} \exp \left[-\frac{\Delta^{2} \tau^{2}}{2}\right] .
$$

Evidently, $\Delta^{-1}=\tau_{\text {mem }}$ : the memory time is given by the inverse coupling width.

Provided the memory time is very small, one can (i) take the Markovian limit, $p(t-$ $\tau) \rightarrow p(t)$, and (ii) do the quasistationary approximation, $\left(t-t_{0}\right) \rightarrow \infty$. Performing the $\tau$-integration in [2] then yields

$$
\dot{p}(t)=-\left(\frac{\alpha^{2} v^{2}}{\hbar^{2} \Delta}\right) p(t)
$$

the usual exponential decay law. The coefficient $\Gamma:=\alpha^{2} v^{2} / \hbar^{2} \Delta$ is called the decay width; it determines the timescale $\Gamma^{-1}=\tau_{\text {rel }}$ on which the expectation value $p(t)$ evolves. Clearly, the Markovian limit is justified only if $\Gamma^{-1} \gg \Delta^{-1}$.

This simple example teaches some important lessons: 
1. The memory time $\tau_{\text {mem }}$ is related to the coupling width: $\tau_{\text {mem }}=\Delta^{-1}$. Hence the memory time is determined by the form of the interaction only; it does not depend on its strength.

2. The relaxation time $\tau_{\text {rel }}$ on the other hand does depend on the strength of the interaction; it is of order $O\left(\alpha^{-2}\right)$ in the coupling constant.

3. Therefore the Markovian limit becomes exact as $\alpha \rightarrow 0$ (while keeping $\Delta, v$ and hence $\alpha^{2} \tau_{\text {rel }}$ fixed - the so-called Van Hove limit [3]).

[1] Nakajima, S., Prog. Theor. Phys. 20, 948 (1958); Zwanzig, R., J. Chem. Phys. 33, 1338 (1960).

[2] Fick, E., Sauermann, G., The Quantum Statistics of Dynamic Processes, Berlin, Heidelberg: Springer 1990.

[3] Van Hove, L., Physica 21, 517 (1955); 23, 441 (1957); Kubo, R., Toda, M., Hashitsume, N., Statistical Physics II, Berlin, Heidelberg: Springer 1985

\subsection{Is Particle Creation Irreversible?}

\section{(J. Rau)}

In the presence of a strong electric field the vacuum becomes unstable; it decays by emitting charged particle-antiparticle pairs, such as electrons and positrons. For the case of spin-1/2 fermions, the number of produced pairs per unit time and unit volume is given by Schwinger's famous formula

$$
w=\frac{(q E)^{2}}{4 \pi^{3}} \sum_{n=1}^{\infty} \frac{1}{n^{2}} \exp \left(-\frac{n \pi m^{2}}{q E}\right)
$$

$[1,2]$. Here $m$ denotes the fermion mass, $q$ its electric charge, and $E$ the magnitude of the external electric field, which is assumed to be time-independent and homogeneous. In a similar manner pair creation occurs in the presence of other fields, such as gauge fields (e.g., chromoelectric fields) or gravitational fields.

This particle creation is perceived as irreversible, despite the fact that the underlying laws of quantum mechanics are invariant under time reversal. In general, such apparent irreversibility is a consequence of a strong separation of time scales and an effective, i.e., coarse-grained description of only the slow degrees of freedom. To understand the apparent irreversibility of particle creation, therefore, it is of great importance to investigate the various time scales exhibited in this physical process.

Mathematically, pair creation is described by a time-dependent Bogoliubov transformation

$$
\mathcal{U}^{(E)}(\tau)\left(\begin{array}{r}
a^{\dagger}(\vec{p}, m) \\
b(-\vec{p},-m)
\end{array}\right)=\left(\begin{array}{r}
\alpha_{\vec{p}}(-\tau) \beta_{\vec{p}}(-\tau) \\
-\beta_{\vec{p}}^{*}(-\tau) \alpha_{\vec{p}}^{*}(-\tau)
\end{array}\right)\left(\begin{array}{r}
a^{\dagger}(\vec{p}-q \vec{E} \tau, m) \\
b(-\vec{p}+q \vec{E} \tau,-m)
\end{array}\right)
$$

mixing positive energy (particle) with negative energy (antiparticle) states. Using the projection method, this tirne-dependent Bogoliubov transformation can be incorporated 
into a kinetic description of an $e^{+} e^{-}$-mixture in the presence of a strong external field, leading to an additional source term in the quantum Boltzmann equation:

$$
\dot{n}_{-}^{\text {source }}(\vec{p}, t)=4 \operatorname{Re} \int_{0}^{t-t_{0}} \mathrm{~d} \tau \dot{\beta}_{\vec{p}}(0) \dot{\beta}_{\vec{p}-q \vec{E} \tau}^{*}(0)\left[\alpha_{\vec{p}}^{*}(-\tau)\right]^{2} S[\vec{p}](t, \tau)
$$

with

$$
S[\vec{p}](t, \tau):=1-\frac{1}{2} n_{-}(\vec{p}-q \vec{E} \tau, t-\tau)-\frac{1}{2} n_{+}(-\vec{p}+q \vec{E} \tau, t-\tau)
$$

being reminiscent of a Pauli blocking factor. This expression is valid in the so-called weak field approximation $\left(\hbar q E \ll m^{2}\right)$. Exploiting the dynamical $S U(2)$ symmetry inherent in the pair creation process [3].

The amplitudes $\dot{\beta}$ and $\alpha$ are determined as

$$
\dot{\beta}_{\vec{p}}(0)=\frac{q E}{2} \cdot \frac{\epsilon_{\perp}}{p_{\|}^{2}+\epsilon_{\perp}^{2}}
$$

and

$$
\begin{aligned}
\alpha_{\vec{p}}^{*}(-\tau) & \left.\rightarrow \alpha_{\vec{p}}^{*}(-\tau)\right|_{\text {adiab }} \\
& =\exp \left[-i \int_{0}^{\tau} \mathrm{d} \tau^{\prime} \omega\left(\vec{p}-q \vec{E} \tau^{\prime}\right)-i \gamma_{\vec{p}}(-\tau)\right] .
\end{aligned}
$$

Here $\gamma$ is the so-called geometric (or Berry's) phase [4].

From the above sorce term c..e can extract both the memory time

$$
\tau_{\mathrm{mem}}=\frac{\epsilon_{\perp}}{q E}
$$

and the time scale $\tau_{\text {prod }}$ on which the relevant observables, i.e., the occupation numbers, evolve. One finds

$$
\frac{\tau_{\text {mem }}}{\tau_{\text {prod }}} \propto \exp \left[-\frac{\pi m^{2}}{2 \hbar q E}\right] .
$$

The particle creation process thus becomes Markovian $\left(\tau_{\text {mem }} / \tau_{\text {prod }} \ll 1\right)$ in the weak field limit, i.e., when $\hbar q E \ll m^{2}$. The Markovian property, in turn, implies an H-theorem and hence is responsible for the perceived irreversibility of particle creation.

[1] J. Schwinger, Phys. Rev. 82, 664 (1951).

[2] W. Greiner, B. Müller, J. Rafelski, Quantum Electrodynamics of Strong Fields, Berlin, Heidelberg: Springer 1985.

[3] A. M. Perelomov, Phys. Lett. 39A, 353 (1972);

Theor. Math. Phys. 19, 368 (1974).

[4] M. V. Berry, Proc. Roy. Soc. A392, 45 (1984). 


\subsection{Vacuum Polarization and Electric Charge of the Positron}

(B. Müller, M.H. Thoma [*])

Hughes and Deutch [1] recently discussed the possibility that the charges of positrons and antiprotons may not be exactly opposite to those of electrons and protons. Whereas the equality in magnitude of the charges of electrons and protons is known to the extreme accuracy $[2,3]\left|Q_{e}+Q_{p}\right|<10^{-21} e$, the equality in magnitude of the charges of electrons and positrons is much more difficult to study directly. After reviewing the available bndy of evidence, Hughes and Deutch conclude that the present limit on the net neutrality of an electron-positron pair is $\left|Q_{e}+Q_{\bar{e}}\right|<4 \times 10^{-8} e$.

There exist far more stringent bounds on this quantity from indirect sources. Our argument is based on the fact that the vacuum polarization in heavy atoms contains an equal number of electrons and positrons and hence would contribute to the overall charge of an atom, if the charges of electrons and positrons do not balance each other precisely. This reasoning is closely related to the observation first made by Morrison [4] and Schiff [5], that the equality of the gravitational masses of electrons and positrons is probed to about 1 percent accuracy by the fact that the contribution of vacuum polarization to the mass of an atom does not lead to a violation of the equivalence principle. This argument is much more powerful in the case of the electric charge. In fact, our bound would be even more precise, would it not be for the necessity of charge renormalization. Since the amount of charge contained in the lowest order (in $Z \alpha$, where $Z$ is the nuclear charge) vacuum polarization is directly proportional to the source charge of the Coulomb field, the net vacuum polarization charge to this order can be absorbed in the renormalized charge of the source, rendering it effectively unobservable. This reasoning does not apply to higher orders in $Z \alpha$ of the atomic vacuum polarization, which do not contribute to charge renormalization.

If the charges of electrons and positrons are not opposite and equal, the first nonvanishing contribution to the overall charge of an atom by the vacuum polarization would come in order $(Z \alpha)^{2}$. According to Furry's theorem, this order normally vanishes identically due to the invariance of quantum electrodynamics (QED) against charge conjugation (C-invariance). However, if $Q_{e}$ and $Q_{\bar{e}}$ do not balance each other, this would iraply a violation of C-invariance and hence invalidate Furry's theorem. For our purposes it is sufficient to consider an effective theory that is consistent at the one-loop level. This is provided by the Lagrangian

$$
L=\bar{\psi}\left(i \gamma^{\mu} \partial_{\mu}-m\right) \psi+\left[Q_{e} \bar{\psi}_{e} \gamma^{\mu} \psi_{e}+Q_{\bar{e}} \bar{\psi}_{\bar{e}} \gamma^{\mu} \psi_{\bar{e}}+\tilde{Q}\left(\bar{\psi}_{e} \gamma^{\mu} \psi_{\bar{e}}+\bar{\psi}_{\bar{e}} \gamma^{\mu} \psi_{e}\right)\right] A_{\mu}
$$

where $\psi_{e / \bar{e}}=P_{ \pm} \psi$ denotes the Dirac field projected on positive and negative energies, respectively, $Q_{e}$ and $Q_{\bar{e}}$ are the charges of electron and positron, and $\tilde{Q}$ denotes the coupling constant associated with pair creation. In addition to $\mathrm{C}$-invariance, the effective Lagrangian breaks gauge and PCT invariance. The violation of PCT invariance is reconciled with the Pauli-Lüders theorem by noting that the projection operators $P_{ \pm}$are nonlocal at the scale of the electron Compton wavelength. 


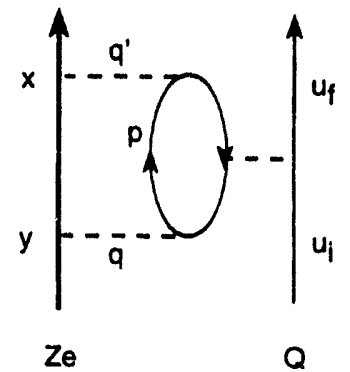

(a)

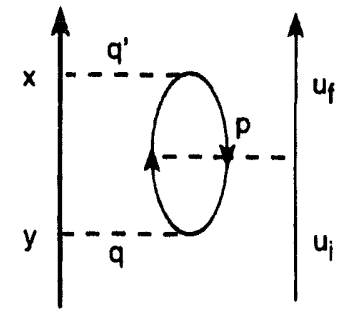

(b)

Fig. 1: Feynman diagrams contributing to second-order (in $Z \alpha$ ) vacuum polarization correction to the forward Coulomb scattering cross section on an atomic nucleus. Diagram (a) is proportional to the positron charge $Q_{\bar{e}}$ and (b) to the electron charge $Q_{e}$.

We consider the limit of forward Coulomb scattering of a nonrelativistic charged particle on the atom, described by the two Feynman diagrams shown in Figure 1. We find that the effect on the scattering amplitude corresponds to the presence of an additional charge

$$
\Delta Z e=\left(Q_{e}+Q_{\bar{e}}\right) Q(Z e \tilde{Q})^{2} \int \frac{d^{3} q}{(2 \pi)^{3}} \frac{F\left(\vec{q}^{2}\right)^{2}}{\vec{q}^{4}} \int \frac{d^{3} p}{(2 \pi)^{3}} \frac{E_{p} E_{p-q}-E_{p}^{2}+\vec{p} \cdot \vec{q}}{E_{p} E_{p-q}\left(E_{p}+E_{p-q}\right)^{2}}
$$

surrounding the nucleus. Here $F\left(q^{2}\right)$ is the nuclear elastic form factor and $E_{p-q}=$ $\sqrt{(\vec{p}-\vec{q})^{2}+m^{2}}$. Since we are not interested in extreme precision, we simply cut off the $q$-integration at the inverse nuclear radius $R$ and evaluate the integrals to leading order in the cut-off. The result is:

$$
\Delta Z e \approx\left(Q_{e}+Q_{\bar{e}}\right) \frac{2 Z^{2} \alpha^{2}}{3 \pi^{2}}\left[\ln \left(\frac{1}{m R}\right)+c\right],
$$

where the constant $c$ depends on the details of the nucleon form factor and can be neglected here. For a heavy atom, such as lead $(Z=82, R=7 \mathrm{fm})$, we find $\Delta Z e \approx \frac{1}{10}\left(Q_{e}+Q_{\bar{e}}\right)$. With the limit on the apparent residual charge of the atom per proton, $\Delta Z \not Z$, we obtain the bound $\left|Q_{e}+Q_{\bar{e}}\right|<10^{-18} e$. Because the net vacuum polarization charge is quadratic in the nuclear charge $Z$, it is impossible to simultaneously adjust the electron-positron and electron-proton charge differences such that all atoms are neutral.

Since the momentum integrations involve only momenta up to $R^{-1}$, and the structure of QED has been tested to very high precision over that range, we believe that our result is essentially model-independent. Because our effective Lagrangian breaks PCT-invariance, our new bound can also be taken as a new test of PCT symmetry, which is better by a factor 4 than the limit derived from the neutral kaon system, but tests a different mode of PCT symmetry breaking.

[*] B. Mülier and M. H. Thoma, Phys. Rev. Lett. 69, 3432 (1992).

[1] R. J. Hughes and B. I. Deutch, Phys. Rev. Lett. 69, 578 (1992).

[2] H. F. Dylla and J. G. King, Phys. Rev. A7, 1224 (1973). 
[3] M. Marinelli and G. Morpurgo, Phys. Lett. 137B, 439 (1984).

[4] P. Morrison, Arn. J. Phys. 26, 358 (1958).

[5] L. I. Schiff, Proc. Nat. Acad. Sci. USA 45, 69 (1959). 


\section{APPENDICES}

\section{IA. Published Articles:}

1. Deterministic Chaos in Non-Abelian Lattice Gauge Theory, B. Müller, A. Trayanov, Phys. Rev. Lett. 68, 3387-3390 (1992).

2. Resolving Minijets in Minimum-Biased Events of Hadronic Interactions, X. N. Wang, Phys. Rev. D46, R1900 (1992).

3. Multiparticle Production in Lepton-Nucleus Collisions and Relativistic String Models, D. J. Dean, M. Gyulassy, B. Müller, E. A. Remler, M. R. Strayer, A. S. Umar, J. S. Wu, Phys. Rev. C46, 2066-2076 (1992).

4. Collective Excitations of the QED Vacuum? Phys. Rev. A47, 854-867 (1993).

5. Vacuum Polarization and the Electric Charge of the Positron, B. Müller and M. H. Thoma, Phys. Rev. Lett. 69, 3432-3434 (1992).

6. Lyapunov Exponent for Classical SU(3) Gauge Theory, C. Gong, Phys. Lett. B298, 257-262 (1993).

7. Strong and Electromagnetic Decays of the Baryon Decuplet, M. Butler, M. Savage, and R. Springer, To appear in Nucl. Phys. B.

8. E2/M1 Mixing Ratio of $\Delta \rightarrow N \gamma$ and Hyperon Resonance Radiative Decay, M. Butler, M. Savage, R. Springer, to appear in Phys. Lett. B.

9. Magnetic Screening in Thermal Gauge theories, T. Biró, B. Müller, To appear in Nucl. Phys. A.

\section{IB. Other Publications:}

1. QCD Vacuum Structure, edited by H. M. Fried and B. Müller, World Scientific (Singapore 1993).

\section{IC. Submitted Articles:}

1. The $\lambda \Phi^{4}$ Theory on the Torus, C. Villarreal, Submitted to Phys. Rev. A.

2. Parton Equilibration in Relativistic Heavy-Ion Collisions, T. S. Biró, E. van Doorn, B. Müller, M. H. Thoma, X. N. Wang, Submitted to Phys. Rev. C. 
3. Probing Hadronic Structure with the Decay $\Delta \rightarrow N \ell^{+} \ell^{-}$, M. Butler, M. Savage, R. Springer, preprint DUKE-TH-93-48.

4. Real-Time Dynamics of Yang-Mills Theories on a Lattice, T. S. Biró, C. Gong, B. Müller, and A. Trayanov, Int. J. Mod. Phys. C (in preparation).

\section{ID. Conference Reports:}

1. Chaotic Dynamics in Non-Abelian Lattice Gauge Theory, C. Gong, B. Müller, A. Trayanov, Third Drexel Symposium on Quantum Nonintegrability, Philadelphia, May 1992.

2. Self-consistent Approximations for Field Theories at Finite Temperature, M. H. Thoma, Workshop on Finite Temperature Field Theory, Winnipeg, July 1992.

3. Physics of the Quark-Gluon Plasma, B. Müller, NATO-ASI on Particle Production in Highly Excited Matter, Il Ciocco, Italy, July 1992.

4. Jets and High Energy Heavy Ion Collisions, X. N. Wang, NATO Advanced Study Institute on Particle Production in Highly Excited Matter, Il Ciocco, Italy, July 1992.

5. Simulations of Ultrarelativistic Heavy Ion Collisions, X. N. Wang, International Conference on High Energy Physics, Dallas, August 1992.

6. On the Metric Structure of Space-Time, J. Rau, Proceedings Salamanca Conference, Salamanca, Spain, June-July 1992.

\section{IIA. Invited Talks:}

1. B. Müller: "Classical Dynamics in Nonabelian Lattice Gauge Theory" (Third Drexel Symposium on Quantum Nonintegrability, Philadelphia, May 1992).

2. M. Thoma: "Stopping Power and Viscosity of the Quark-Gluon Plasma" (Phenomena at High Temperature and Density-Thermal Field Approach, Bielefeld, Germany, May 1992).

3. B. Müller (Organizer): "QCD Vacuum Structure and Its Applications" (Workshop, American University of Paris, June 1992).

4. J. Rau: "On the Origin of the Metric Structure of Space-Time" (XIX International Colloquium on Group Theoretical Methods in Physics, Salamanca, Spain, July 1992).

5. B. Müller: "Physics of the Quark-Gluon Plasma" (NATO Advanced Study Institute on Particle Production in Highly Excited Matter, Il Ciocco, Italy, July 1992).

6. X. N. Wang: "Jets and High Energy Heavy Ion Collisions" (NATO Advanced Study Institute on Particle Production in Highly Excited Matter, Il Ciocco, Italy, July 1992).

7. M. Thoma: "Hartree Approximation for QCD at Finite Temperature" (Perturbative Methods in Hot Gauge Theories, Winnipeg, Canada, July 1992). 
8. X. N. Wang: "Simulations of Ultrarelativistic Heavy Ion Collisions" (XXVI International Conference on High Energy Physics, Dallas, TX, August 1992).

9. B. Müller: "Exciting Physics with STAR" (STAR Collaboration Meeting, Brookhaven, September 1992).

10. A. Trayanov: "Computational Aspects of Real-Time Evolution in Lattice Gauge Theory" (Fifty-Ninth Meeting of the SESAPS, Oak Ridge, November 1992).

11. R. Springer: "B Physics at the SSC" (SSC Symposium, Madison, WI, March 1993).

\section{IIB. Contributed Talks and Seminars:}

M. Thoma: "Stopping Power and Viscosity of the Quark-Gluon Plasma" (University Munich, May 15, 1992).

M. Thoma: "Small Violations of the Pauli Exclusion Principle" (Technical University, Munich, June 4, 1992).

J. Rau: "On the Origin of the Metric Structure of Space-Time" (University of Debrecen, Hungary, June 5, 1992).

X. N. Wang: "Pre-equilibrium Scattering in Heavy Ion Collision" (A Workshop: QCD-20 Years Later, Aachen, June 9-13, 1992).

J. Rau: "Nonequilibrium Stat. Quantum Field theory" (Central Research Institute for Physics, Budapest, Hungary, June 11, 1992).

J. Rau: "On the Origin of the Metric Structure of Space-Time" (NATO Advanced Study Institute on Recent Problems in Mathematical Physics, Salamanca, Spain, June $25,1992)$.

X. N. Wang: "Hard processes in Heavy Ion Collisions" (CERN, June 25, 1992).

X. N. Wang: "High $p_{T}$ Probes in Heavy Ion Collisions" (Institute for Nuclear Physics, Frankfurt, July 2, 1992).

X. N. Wang: "Quark Gluon Plasma or Mini-jets?" (University of Regensburg, July $7,1992)$.

B. Müller: "Chaos in Nonabelian Lattice Gauge Theory" (University of Minnesota, Minneapolis, September 25, 1992).

M. Thoma: "Finite Temperature Field Theory and Applications to the Quark-Gluon Plasma" (University Giessen, 3 lectures).

R. Springer: "Nuclear Parity Violation and Strangeness in the Nucleon" (North Carolina State University, October 1992).

B. Müller: "Relativistic Nucler Collisions and Quark-Gluon Plasma" (N.C. State University, Colloquium, Raleigh, November 9, 1992). 
R. Springer: "Heavy Baryon Chiral Perturbatiion Theory and Decays of the Baryon Decuplet" (Colloquium, University North Carolina, November 1992).

M. Thoma: "Thermalization of the Quark-Gluon Plasma in Ultrarelativistic Heavy Ion Collisions" (University Ciessen, December 17, 1992).

J. Rau: "The Entropy Problem in Quantum Field Theory" (University of Heidelberg, Germany, December 21, 1992).

M. Thoma: "Damping Rates and Equilibraiion of the Quark-Gluon Plasma" (Gross Properties of Nuclei and Nuclear Interactions XXI, Hirschegg/Austria, January 20, 1993).

Müller: "Relativistic Nuclear Collisions: Probing the Early Universe in the Laboratory" (McGill University, Montreal, Canada, February 11, 1993).

R. Springer: "Weak and Strong Decays of the Decuplet of Baryons" (Massachusetts Institute of Technology, February 1992).

B. Müller: "Vacuum Polarization and Electric Charge of the Positron" (INT, Seattle, March 8, 1993).

B. Müller: "Chaos in Nonabelian Lattice Gauge Theory" (Brown University, March 15, 1993).

B. Müller: "Relativistic Heavy Ion Collisions: Probing the Early Universe in the Laboratory" (Brown University, March 15, 1993).

B. Müller: "The Creation of the Quark-Gluon Plasma" (LNS Colloquium, MIT, Cambridge, March 16, 1993).

B. Müller: "Formation of the Quark-Gluon Plasma" (Cyclotron Colloquium, MSUNSCL, March 17, 1993).

B. Müller: "Chaos in Nonabelian Gauge Theories" (Michigan State University, March 18, 1993).

M. Thoma: "Parton Equilibration in Relativistic Heavy Ion Collisions" (German Physical Society, Mainz, March 23, 1993).

B. Müller: "Relativistic Heavy Ion Collisions: Probing the Early Universe in the Laboratory" (University of Oregon. Eugene, April 29, 1993).

\section{Triangle Nuclear Theory Colloquium Series}

9/8/92 Vitaly Efimov (University of Washington)

Threshold three-Body Effects: Physics and Applications

9/15/92 Manoj Banerjee (University of Maryland)

A Quark Model of the Nuclecn

9/22/92 Mark Wise (California Institute of Technology) 
New Symmetries of the Strong Interaction

10/6/92 Carl Dover (Brookhaven National Laboratory \& Yale University) Strange Quarks and Nucléi

10/20/92 Anatoly Radyushkin (Old Dominion University)

QCD Sum Rules and Heavy Quarks

10/27/92 Martin Savage (University of California, San Diego)

Theoretical Aspects of Nuclear Parity Violation

11/3/92 Dan Riska (University of Helsinki)

Hyperon Physics

11/5/92 Dirk Walecka (College of William and Mary)

Overview of the CEBAF Scientific Program

11/10/92 Gerald Miller (University of Washington)

Color Transparency and Nuclear Phenomena

11/17/92 A. Baha Balantekin (University of Wisconsin-Madison)

Theoretical Status of the Solar Neutrino Problem

12/4/92 Nikola Cindro (Rudjer Boskovic Institute)

Measuring the Very Large and the Very Small Determination

of the Reaction Zone in Nuclei by Astrophysical Methods

12/8/92 Fritz Coester (Argonne National Laboratory)

Light-Front Dynamics of Nucleons and Quarks

1/12/93 Jorge Piekarewicz (SCRI, Florida State University)

Parity Violation in Electron Scattering

1/19/93 Carlo Carraro (Harvard University)

Final State Interactions in Quasielastic Scattering

1/26/93 Alexander Bochkarev (University of Pittsburgh)

Diffusion Rate in Field Theory at High Temperature

2/2/93 Jonathan Engel (Bartol Research Institute)

Double Beta Decay and Nuclear Theory

2/16/93 Tamas Biró (Universität Giessen, Germany)

Transport Theories of Heavy-Ion Reactions

2/23/93 Steven Pollack (NIKHEF, Amsterdam)

Effects of Particle and Nuclear Physics in Atomic Parity Violation

3/2/93 Scott Chapman (University of California, Berkeley)

An Introduction to Nonabelian Monopoles

3/9/93 Adam Szczepaniak (North Carolina State University)

Effective Degrees of Freedom in Hadonic Structure 
3/16/93 Eric Swanson (Massachusetts Institute of Technology)

Hadronic Interactions from the Constituent Quark Model

3/23/93 Ming Chu (California Institute of Technology)

Quark Correlation Functions in Lattice QCD

3/30/93 David Richards (University of Edinburgh, (UKQCD)

Recent Lattice Results on the Spectrum and Heavy Quark Physics

4/6/93 Colin Morningstar (SLAC)

Nonrelativistic Lattice - QCD

4/15/93 Pankaj Jain (University of Kansas)

$Q \bar{Q}$ Bound States in the Bethe-Salpeter Formalism

4/20/93 Stefan Schramm (Indiana University)

Hadrons and the QCD Vacuum in a Phenomenological Model

4/22/93 Avaroth Harindranath (Ohio State University)

Light-front Approach to the Relativistic Bound State Problem

\section{Theory Seminars at Duke 1992/93}

5/7/92 Gerhard Soff (GSI Darmstadt, Germany)

New Particle Production by Heavy Ions Supercollider Energies

6/18/92 Eric van Doorn (Duke University)

Coalescence Model and Formation of Meson-Meson Atoms

in Relativistic Heavy-ion Collisions

6/19/92 Carsten Greiner (University of Erlangen)

Memory Effects in Relativistic Transport Theory

6/24/92 Markus Thoma (TU München and Duke University)

Self-consistent Approximations for Field Theories at Finite Temperature

6/23/92 David Neu (University of California, Berkeley)

The Coulomb Singularity Revisited

8/12/92 Markus Thoma (TU München and Duke University)

Finite Temperature Field Theory (5 lectures)

9/9/92 Markus Thoma (Duke, TU München, Giessen)

Violations of the Pauli Principle?

9/11/92 Tamás Biró (Universität Giessen)

Nuclear and Quark Transport Models

9/16/92 Manoj Banerjee (University of Maryland)

Role of Instantons in a Chiral Confining Model

9/23/92 Mark Wise (California Institute of Technology) 
Chiral Perturbation Theory

9/30/92 Tamás Birć (Universitaet Giessen/Duke)

Magnetic Monopoles in Hot QCD

10/7/92 Joshua Socolar (Duke University)

The Mysteries of Self-organized Criticality

10/7/92 Carl B. Dover (Brookhaven National Laboratory)

Antimatter Interacting with Matter

10/12/92 Malcolm Butler (Queens University of Canada)

The Electromagnetic Polarizabilities of the Nucleon

10/14/92 Martin Savage (University of California, San Diego)

A QCD Calculation of the Binding of Quarkonium in Nuclear Matter

10/21/92 Anatoly Radyushkin (C.E.B.A.F.)

Hadronic Form Factors

10/28/92 Robert Brown (Duke University)

Long Range Order in Two Dimensions?

11/4/92 Robert Brown and J. Socolar (Duke University)

Order in 2nd lattices and $\mathrm{O}(3)$ Continuous Hamiltonians

- The Mermin-Wagner Theorem

11/11/92 Charles Evans (Univerity of North Carolina)

Critical Phenomena in Black Hole Formation

11/25/92 Henry Greenside (Duke University)

Models for space-Time Chaos

11/30/92 Berndt Müller (Duke University)

Disoriented Chiral Condensates

12/2/92 Jianping Lu (University of North Carolina)

The Structural and Electronic Properties of Fullerenes

and Fullerides-the Effect of Orientational Dis/order

12/15/92 Paul Coddington (Syracuse Univerity)

Parallel Computing Lattice Gauge and Spin Models

12/16/92 E. David Davies (University of Stellenbosch \& TUNL)

Chaos and Time Reversal

2/3/93 Wei Lin (University of Washington, Seattle)

Singularity Structure of Hadronic Form Factors

2/8/93 Carsten Greiner (Duke University)

Strange Quark Matter in Relativistic Heavy Ion Collisions

2/17/93 Henry Greenside (Duke University) 
Anderson vs. Weinberg or Is Simplicity Beautiful?

2/22/93 Dimitri Kalafatis (IPN Orsay)

Nonlinear Realizations of Chiral Symmetry and Topological Soliton Stability

2/24/93 James D. Bjorken (Stanford Linear Accelerator Center)

A Full-Acceptance Detector for the SSC

3/1/93 Anand Subbaraman (Syracuse University)

Chiral Interactions of Heavy Mesons

3/1/93 Berndt Müller (Duke University)

Vacuum Polarization and the Positron's Charge

3/3/93 Michael Prisant (Department of Chemistry, Duke University) Applications of Ray Casting on a Massively Parallel Computer to Protein Structure

3/5/93 Derek Leinweber (University of Maryland)

What does Lattice QCD Teach us about the Electromagnetic Structure of Baryons

3/8/93 Jochen Rau (Duke University)

Remarks on Entropy, Irreversibility, and the Quantum Boltzmann Equation

3/22/93 Sergei Matinian (Duke University and Yerevan)

Branching Processes and Multiple Production

3/24/93 Horst Meyer (Duke University)

Equilibration Studies Near the Liquid-Vapor Critical Point:

Relevance to the N.A.S.A. Low Gravity Program

3/26/93 Sen-Ben Liao (Massachusetts Institute of Technology)

Blocking Transformations in Field Theory

4/7/93 Adam Szczepaniak (North Carolina State University)

Effective Degrees of Freedom in Mesonic Structures 

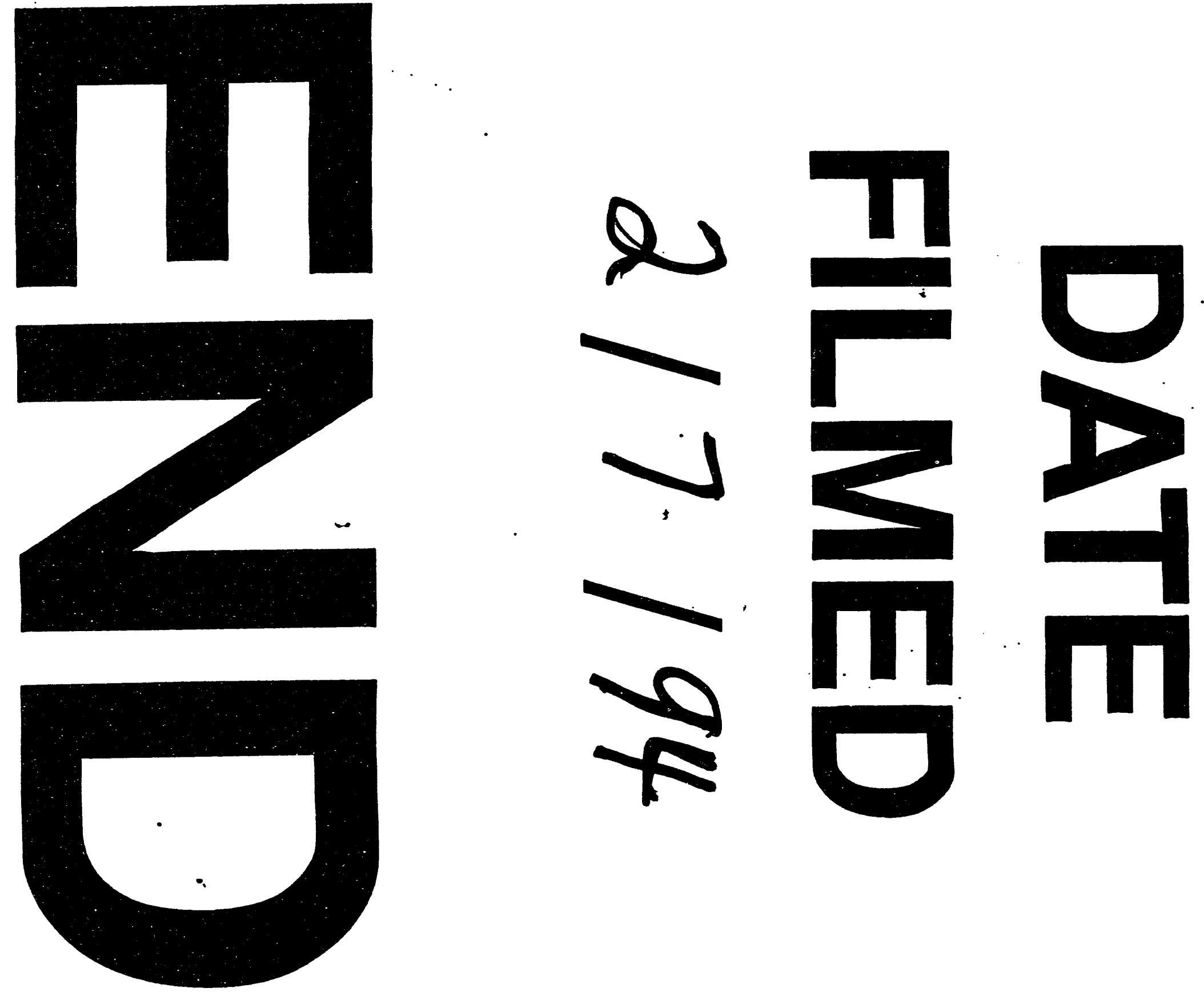
1 